\title{
SCANDI - an all-sky Doppler imager for studies of thermospheric spatial structure
}

\author{
A. L. Aruliah, E. M. Griffin, H.-C. I. Yiu, I. McWhirter, and A. Charalambous \\ Atmospheric Physics Laboratory, University College London, Gower Street, London WC1E 6BT, UK \\ Received: 15 July 2009 - Revised: 12 January 2010 - Accepted: 24 January 2010 - Published: 15 February 2010
}

\begin{abstract}
A new all-sky Fabry-Perot Interferometer called the Scanning Doppler Imager (SCANDI) was built and installed at Longyearbyen in December 2006. Observations have been made of the Doppler shifts and Doppler broadening of the $630 \mathrm{~nm}$ airglow and aurora, from which upper thermospheric winds and temperatures are calculated. SCANDI allows measurements over a field-of-view (FOV) with a horizontal radius of nearly $600 \mathrm{~km}$ for observations at an altitude of $250 \mathrm{~km}$ using a time resolution of $8 \mathrm{~min}$. The instrument provides the ability to observe thermospheric spatial structure within a FOV which overlaps that of the EISCAT Svalbard radar and CUTLASS SuperDARN radars. Coordinating with these instruments provides an important opportunity for studying ion-neutral coupling. The all-sky image is divided into several sectors to provide a horizontal spatial resolution of between $100-300 \mathrm{~km}$. This is a powerful extension in observational capability but requires careful calibration and data analysis, as described here. Two observation modes were used: a fixed and a scanning etalon gap. SCANDI results are corroborated using the Longyearbyen single look direction FPI, and ESR measurements of the ion temperatures. The data show thermospheric temperature gradients of a few Kelvins per kilometre, and a great deal of meso-scale variability on spatial scales of several tens of kilometres.
\end{abstract}

Keywords. Atmospheric composition and structure (Airglow and aurora) - Meteorology and atmospheric dynamics (Thermospheric dynamics; Instruments and techniques)

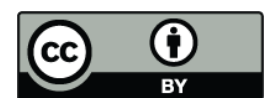

Correspondence to: A. L. Aruliah (a.aruliah@ucl.ac.uk)

\section{Introduction}

The upper thermosphere has a high viscosity because it is a tenuous gas; for example, at an altitude of $300 \mathrm{~km}$ it has a density of the order of $10^{14}-10^{15}$ particles $\mathrm{m}^{-3}$. High viscosity smoothes out small-scale structures so that the upper thermosphere is expected to have large spatial scales of around a thousand kilometres and temporal scales of a few hours to respond to ionospheric forcing (e.g. Rishbeth and Garriot, 1969). However, for many years FPIs have provided direct measurements of the upper thermosphere that have shown meso-scale behaviour achieved using single FPIs, bistatic and tristatic FPI arrangements with single point measurements (e.g. Biondi et al., 1984; Greet et al., 1999; Aruliah et al., 2005, respectively). Attempts have been made to image the whole sky simultaneously using fixed gap etalons (e.g. Rees et al., 1984), but the detector technology at that time made it very difficult. The best detector of the 1980s was an Imaging Photon Detector which had a quantum efficiency in the range 10-15\% (McWhirter et al., 1982). The IPD's resistive anode and its associated electronics had limited stability and tended to drift with time so that the mapping of the sky onto the detector was not reliable enough for the high precision required. Consequently there are few papers in existence compared with the FPI workhorse (e.g. Batten and Rees., 1990; Biondi et al., 1995).

In the mid-1990s Conde and Smith built an innovative allsky FPI that they called the Scanning Doppler Imager (SDI) (Conde and Smith, 1997). This was installed at Poker Flat in Alaska and vividly demonstrated the dynamic response of the thermosphere to auroral forcing (Conde et al., 2001). Based on this concept, the Atmospheric Physics Laboratory deployed a new all-sky Fabry-Perot Interferometer called the Scanning Doppler Imager (SCANDI) at Longyearbyen in December 2006. Observations have been made of the Doppler shifts and Doppler broadening of the $630 \mathrm{~nm}$ airglow and aurora, from which winds and temperatures are

Published by Copernicus Publications on behalf of the European Geosciences Union. 


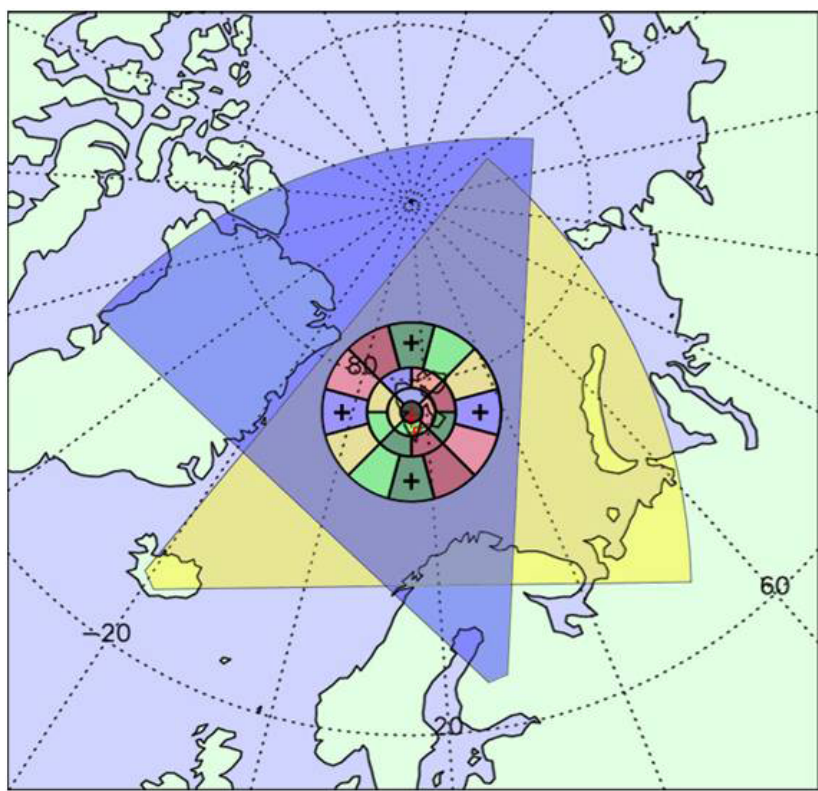

Fig. 1. The SCANDI field-of-view overlaps that of the ESR and SPEAR radars, and is also within the fov of the CUTLASS radars. The radars measure ionospheric parameters while SCANDI measures co-located thermospheric parameters.

calculated. The peak emission height is around $240-250 \mathrm{~km}$ at this latitude. SCANDI has a $140^{\circ}$ field-of-view which translates to a radius of $\sim 565 \mathrm{~km}$ for this emission height. The location of Longyearbyen means that the SCANDI fieldof-view passes under the cusp. It also covers the boundary between the polar cap and auroral oval, and thus the region between open and closed field lines, which makes it a very interesting location in which to study upper atmosphere dynamics and energetics. The latitude allows $24 \mathrm{~h}$ of nighttime observation between December and January each winter, which is necessary for optical measurements of aurora and airglow. SCANDI is also within the viewing fields of the CUTLASS radars and directly overlaps the SPEAR and ESR radar fields-of-view, as shown in Fig. 1. Thus it is now possible to provide studies of ion-neutral coupling over an extended area, using independent measurements of the ionosphere and thermosphere.

\section{SCANDI instrument}

The original concept for a scanning all-sky FPI comes from Conde and Smith (1997). Their instrument powerfully extends the capability of the single look direction FPI which uses a $1^{\circ}$ field-of-view. Early FPIs used for upper atmosphere studies had photometers as detectors, with integration times of around $5 \mathrm{~min}$, which was necessary for the very low emission intensities typical at low- and mid-latitude sites (e.g. Biondi et al., 1984; Meriwether et al., 1983; Burnside et al., 1981). In the 1980s the APL developed a state-of-theart Imaging Photon Detector, which was produced with both S20/25 and GaAs photocathodes. The ratios of the charge pulses from the position-sensing resistive anode were processed to give a 2-dimensional image array, initially recorded as a $64 \times 64$ pixel image and ultimately as $256 \times 256$ pixels (Rees et al., 1980). The excellent signal-to-noise (s/n) ratio inherent in this photon-counting system allowed exposure times of only $60 \mathrm{~s}$ from high-latitude sites, where auroral activity produces emissions at least an order of magnitude greater than those produced at mid- and low-latitudes. Over the last 20 years, advances in Charge Coupled Device (CCD) technology have produced detectors which have surpassed the IPD in Detected Quantum Efficiency (DQE) but only relatively recently, with the development of the Electron Multiplying CCD (EMCCD) has the combination of high DQE, high $\mathrm{s} / \mathrm{n}$ ratio and high-speed readout produced an imager that can equal the low-noise performance and high-speed readout of the IPD whilst providing the high DQE, image stability and positional linearity of the CCD. This combination, coupled with a robustness not obtainable with photocathode technology, has provided the enabling technology for a new generation of all-sky FPIs (McWhirter, 2008).

The FPI uses a mirror tilted at a particular elevation angle (e.g. $30^{\circ}$ for the Svalbard FPI) that turns to point in different directions. A typical sequence of FPI look directions is: to the north, east, south, west, zenith and a calibration lamp. The reason for rotating through cardinal look directions is that a wind vector can be derived by using 2 orthogonal look directions (e.g. north and east); or using the average of the north and south, and applying a vector addition to the average of the east and west look directions; or by fitting a sinusoidal function to all the available look directions. The validity of this process requires 4 major assumptions:

- The emission height is constant over the field-of-view.

- The wind is uniform over the field-of-view.

- The wind remains largely unchanged for the duration of a full cycle of look directions.

- The vertical wind component is zero or small enough compared with the horizontal component to be ignored.

Note that for a $250 \mathrm{~km}$ peak emission height the horizontal diameter of the field-of-view (FOV) is several hundred kilometres depending on the elevation angle used for the FPI look direction. Consequently this is a perfectly adequate procedure for generating the large-scale behaviour of thermospheric winds. It is also in keeping with the concept of a highly viscous medium, with minimal variability and shear. The two major 3-dimensional coupled thermosphereionosphere global circulation models that arose in the 1970s1980s used coarse grids for the thermosphere, partly limited by computational power and partly because it was thought unnecessary to provide a higher spatial resolution for the 


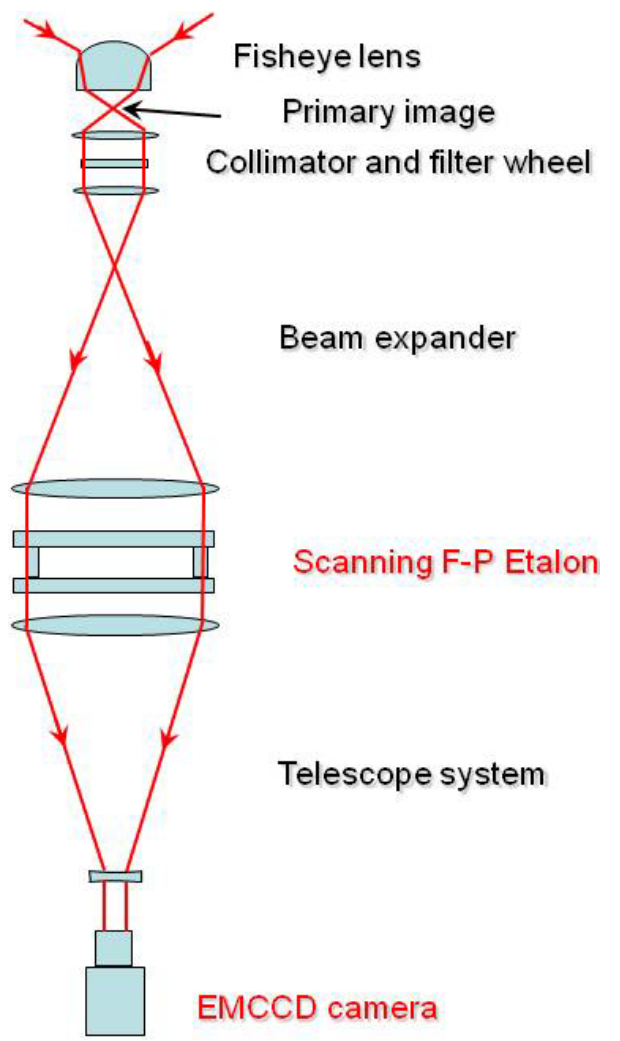

Fig. 2a. The Scanning Doppler Imager. Simplified optics (not-toscale).

thermosphere (e.g. Fuller-Rowell and Rees, 1980; Roble et al., 1983). These models provided valuable insights into the large scale circulation of the upper atmosphere. However, since computing power is no longer a limiting factor, recent observations of meso-scale structure now provide the incentive to improve the spatial resolution of thermospheric models.

The SCANDI provides observations of meso-scale structure from all-sky images of the wind, temperature and $630 \mathrm{~nm}$ intensity field. Measurements are made in all directions at the same time. In theory the number of look directions can be as large as the number of detector pixels. However, balancing the necessity of maximising the photon count and also maximising the spatial resolution requires the fieldof-view to be divided into sectors. Currently this is a period of solar minimum and there are 25 sectors, but it is possible to use a larger number to increase spatial resolution for periods when the intensities are higher (Sect. 2.3.2) such as during particle precipitation and during solar maximum. Conde et al. are running their latest SDI with 115 sectors (private communication).

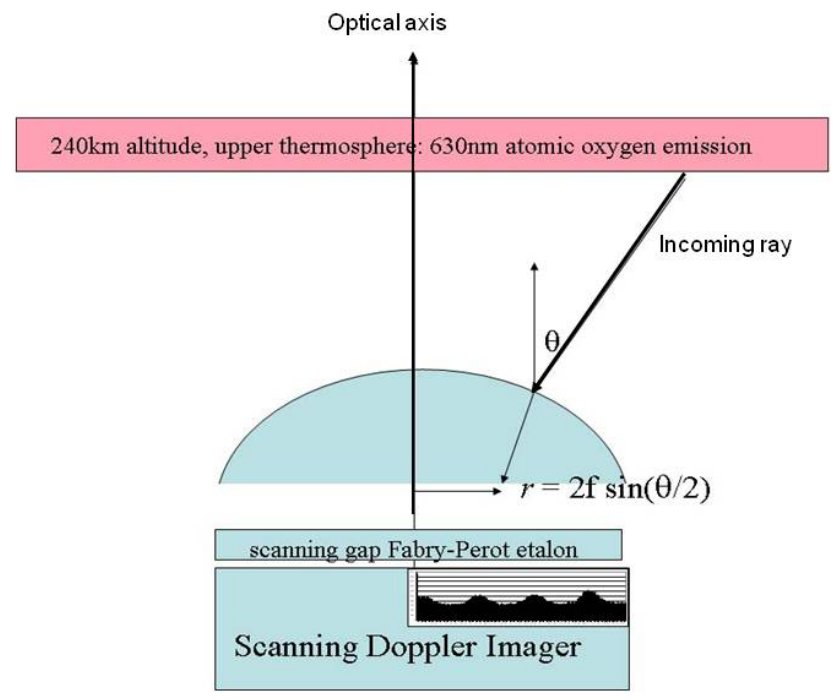

Fig. 2b. The equisolidangle fish-eye lens projection in SCANDI.

\subsection{Design and construction of SCANDI hardware}

In order to obtain the maximum mechanical stability, it was decided to adopt an approach of "alignment by design", whereby the optics of the entire instrument were modelled by computer, after which the positions of the optical components were fixed, with only minimal adjustment of focal distances available in the finished instrument. The instrument is physically very long $(3 \mathrm{~m})$. To minimise flexure, a cylindrical construction was adopted for most of the optical path. The entire optical system is temperature controlled to maintain image stability. The instrument is mounted on a custom-made height-adjustable floor-standing framework which facilitates installation in different locations.

\subsubsection{Optical configuration}

Figure 2a shows a schematic plan of SCANDI. The instrument consists of five sections, each of which is described below.

\section{The all-sky imaging lens}

This is a commercial fisheye lens (Sigma, $f=8 \mathrm{~mm}$ ) with a nominal equi-solid angle projection.

\section{Filter collimator and calibration unit}

The light from the primary image of the all-sky lens is collimated into a $50 \mathrm{~mm}$ diameter light path, where it passes through one of the $2^{\prime \prime}$ narrow-band interference filters set in a four position filter wheel. One position is left empty to allow broad-band imaging. The $630.0 \mathrm{~nm}$ and $557.7 \mathrm{~nm}$ filters have $1 \mathrm{~nm}$ bandwidth. The filter is overfilled with illumination according to good optical design practice. The central 
wavelength of the filter is made to be $0.2 \mathrm{~nm}$ higher than the emission wavelength, and the wave path design ensures that all light rays are contained within a sufficiently narrow cone angle so that all are within the passband. Thus there is no significant reduction of transmission towards the edge of the filters. Prior to the filter wheel, a $45^{\circ}$ mirror may be rotated into the optical path to obscure the sky image and direct light into the system from a $3^{\prime \prime}$ square fibre-optic panel, into which light from the spectral calibration sources is injected.

\section{Beam expander}

The emerging beam is then expanded to $150 \mathrm{~mm}$ diameter to match the aperture of the etalon. The resultant reduction in the light-cone angle means that the entire field of view is mapped onto five orders of interference of the etalon, corresponding to a cone angle of approximately $3^{\circ}$.

\section{Capacitance Stabilised Etalon (CSE)}

The CSE was designed and built at APL and consists of a Fabry-Perot etalon with the three conventional fixed spacers separating the two parallel mirrors replaced by piezo-electric transducers. In order to control the cavity length accurately, the plate separation at each spacer is monitored with a capacitance sensor. A three channel servo loop incorporates sensitive capacitance bridge circuits and high voltage amplifiers, the outputs of which control the length of the piezo-electric spacers. The spacer length is varied under computer control by the application of a small offset to the bridge. The etalon is contained in a sealed, temperature controlled housing which is mounted on a three-point support to allow tilting of the etalon in order to centre the fringe pattern on the final image.

\section{Beam reducing telescope and imager}

The final optics consist of a Galilean beam reducer and a fast $(f / 1.8)$ commercial camera lens, which focuses the final image onto a state-of-the art Electron Multiplying CCD camera (Andor DV887).

\subsubsection{Optical alignment}

The optics may be considered as the superposition of two separate systems: an all-sky imager and a Fabry-Perot interferometer. Each has to be aligned and focussed independently to achieve the final image. By rotating the $45^{\circ}$ mirror into the path, laser light is injected into the filter collimator to provide stable Fabry-Perot fringes, which are focussed and centred on the camera. Focussing is achieved by adjustment of the final camera lens, and centring of the fringe pattern is achieved by adjustment of the three support points of the etalon. The optics are then operated in broad-band mode, with the narrow-band interference filter rotated out of the light path. In this configuration the instrument functions solely as an imager and the sky image can be centred and focussed. Centring is achieved by replacing the field lens at the primary image plane of the fisheye lens with a central pinhole aperture. The lateral position of the lens is then adjusted to centre the pinhole in the camera image.

\subsection{SCANDI control software}

In its simplest form, a Doppler imager can be realised by using Fabry-Perot etalon fringes (i.e. the Airy function) to modulate an all-sky image. A conventional FPI measures the shift in radius of the fringe (by a few pixels), which is superimposed on a small area of sky (typically $1^{\circ}$ field-of-view). For a Doppler imager with a fixed etalon gap, several fringes are mapped onto the whole sky and Doppler shifts are determined by measuring the radial displacement of segments of the ring pattern (Batten and Rees, 1990). Here the winds and temperatures can be found only for those segments of the sky that correspond to peaks in fringe intensity. Another limitation of this method is that itis subject to large errors because the radial shift in the outermost fringes is very small - only a fraction of a pixel - and consequently depends on defining and maintaining the precise centre of the ring pattern. The Conde and Smith method elegantly avoids this drawback by changing the etalon gap of a CSE to scan the fringe pattern across the whole sky image so that every part of the sky will be mapped to a peak fringe intensity at some point of the scan. The etalon gap is increased to allow for exactly one free spectral range (FSR) in, typically, 120 steps and an image is recorded at each step. Thus each pixel of the image acts as a separate point detector and creates its own interferogram when all 120 steps are considered.

The system is calibrated by scanning whilst illuminated with a stabilised single-frequency laser. The step position which corresponds to the peak fringe intensity response is determined for each pixel and stored in the "phase map" lookup table. The concentric areas represent successive orders of interference. The laser look-up table is then generalised by removing any offset from zero and extrapolating the Airy function by adding $2 \pi$ before and after the stored intensities in these 120 steps. New phase map look-up tables for the $630.0 \mathrm{~nm}$ sky signal and $630.48 \mathrm{~nm}$ neon calibration lamp are then created by multiplying by a factor equal to the ratio of the emission wavelength to the laser wavelength. The phase maps are finally re-segmented into concentric sections by subtracting multiples of the appropriate FSR to return to a single FSR per 120 steps for each wavelength. The procedure is explained in detail in Conde and Smith (1997).

A sky image sequence is then recorded in the following manner: the sky is divided into zones; the intensities from the pixels in each zone are added together in order to obtain a satisfactory signal to noise ratio. At each step, the signal from each pixel is added into the composite interferogram for its zone according to the step number in the scan and the phase adjustment indicated by the phase map. This 
is explained fully in Conde and Smith (1997). The system is self-calibrating and does not require a precise centre to be defined. However, it does require that the etalons are strictly parallel and remain parallel throughout the scanning procedure. Consequently the gain on the three piezo-electric transducers must be identical. Slight deviations from this ideal situation result in further calibrations being required as described in Sect. 2.4.4.

The etalon gap is stepped up in size as fast as possible in order to minimise the distortion to the Airy function of temporal changes in intensities, winds and temperatures during the period of a complete scan. The entire scan is repeated a few times until sufficient photons have been gathered. The system therefore requires the co-addition of several hundred images from each zone, since each pixel produces its own Airy function. This is made possible by the use of an electron-multiplying charge coupled device (EMCCD) which, unlike a conventional CCD, can be read out very quickly, typically less than $30 \mathrm{~ms}$, without compromising the signal-to-noise ratio. Stringent demands are placed upon the software to minimise the processing time for each image, during which each pixel has to be dark-field corrected and allocated to the correct point in the interferogram whilst the etalon is stepped to the next position. Currently, a single scan takes around 1-2 min in order to give a good compromise between maintaining a fast scan and maximising the ratio of exposure time to processing time. Three to five scans must be added together to allow a representative sampling of the whole of the Airy function during the typical 7-8 min exposure. This is especially important for observations made at auroral latitudes when there can be considerable intensity fluctuations during a scan. Noise spikes due to cosmic rays are automatically excluded by discriminating against events bigger than a predefined threshold.

The instrument is controlled by a fully integrated program written in Delphi which incorporates the EMCCD camera control and control modules for the CSE, calibration lamp, laser, calibration source mirror and filter wheel. The desired sequence of events is written in the form of a text file in which the various functions are expressed as single letter commands which set exposure time, create the phase maps, perform the sky scan and control the filter wheel, calibration lamps and laser. Full control of all functions is also available manually from the keyboard. An on-screen plot of radial distance squared versus intensity facilitates precise alignment of the etalon. An almanac module ensures that data is taken only during the desired hours of darkness (currently, when the solar zenith angle is greater than $96^{\circ}$ ).

\subsection{Sky mapping}

\subsubsection{Fish-eye lens projection onto detector}

SCANDI uses a fish-eye lens in its all-sky imaging optical system to project a $150^{\circ}$ field-of-view onto the EMCCD detector. There are various types of manufactured fish-eye lenses available in the market and their relative performance can be found in Kulmar and Bauer (2000). An equi-solidangle projection fish-eye lens was chosen for SCANDI, and each incoming ray can be mapped onto an output image using Eq. (1).

$r=2 f \sin (\theta / 2)$

Where $r$ is the radial distant between the image point and the optical axis, $f$ the fish-eye lens focal length, and $\theta$ the angle between the incoming ray and the optical axis (Fig. 2b). Since SCANDI has a vertical optical axis, $\theta$ is effectively the zenith angle. To allow proper projection of SCANDI data to the thermosphere it is necessary to quantify how an all-sky image is translated through SCANDI's optics onto the detector, and to allocate the corresponding azimuth and elevation observational angles to each CCD pixel and SCANDI zone.

There are two possible sources for the projection calibration curve: the detected moon and star-field positions. Both can be compared against the corresponding locations obtained from an almanac or star charting software such as Skymap (www.skymap.com). Both sources are tested and described in this section. The equi-solid-angle model (Eq. 1) alone is not sufficient to represent the whole projection system, which is an ideal derivation assuming reflection from a reflective sphere. All real lenses deviate from it by a few percent. Furthermore, there are other optics between the fisheye lens and the detector that need to be considered. Hence Eq. (1) is used as a guideline only, and instead a calibration projection curve is independently fitted to determine the precise parameters.

\section{Centring}

The zenith of the sky image does not lie exactly in the centre of the EMCCD detector. The star charting software can be used to determine star positions as the star field rotates through the night and by comparing these with $(x, y)$ coordinates measured on the EMCCD image, the exact location of the celestial zenith on the image, $\left(x_{o}, y_{o}\right)$ can be determined.

This centre check is performed on both the moon and star data (described in the following section) before the curve fitting described in the following sections, and the best centre resulting from the star data is determined.

\section{The moon and star-field}

An empty location in the filter wheel enables the instrument to be operated in broad-band mode. This means that the moon and star-field, which are quasi-white light sources, can be imaged without the added modulation of the Fabry-Perot rings. The moon was found to be unsuitable as a source for mapping the pixels onto the sky. The shape of the moon was distorted into a crescent by the mapping of the lens. The 


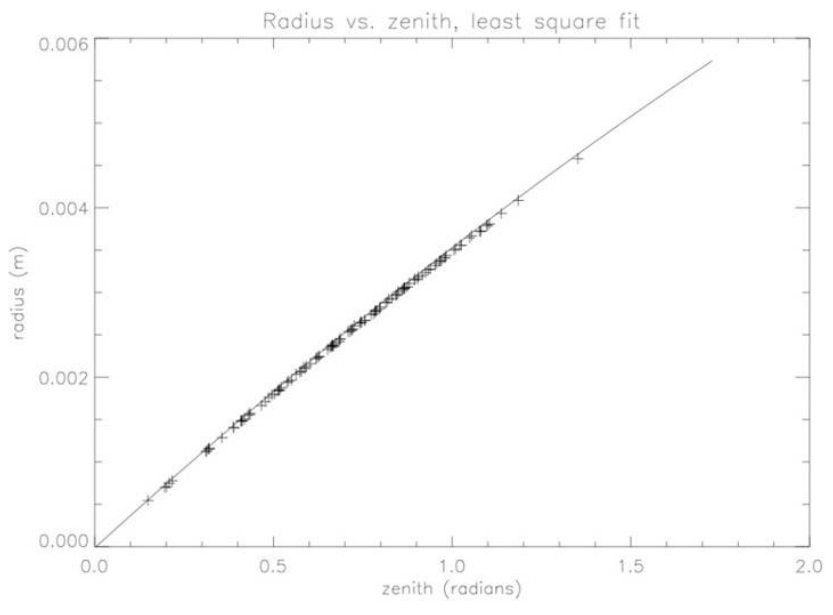

Fig. 3. The least square fit (solid line) on the plot of radius (from the optical axis on the CCD) versus zenith angle (obtained from sky map).

bright spot spanned across tens of pixels, covering a small range of azimuths $\left(120^{\circ}\right)$ and elevations $\left(11^{\circ}\right)$. It was therefore difficult to pinpoint the exact moon centre. A far better mapping was provided by the star field.

Star-field images were constructed for 21 January 2007, when the sky was mostly free of cloud. The star field was clearly visible from most images, allowing a high number of calibration points, with a good range of elevation and azimuth angles, and 125 star locations were collected for the projection fit. Two equations were fitted for comparative purposes: a least squares fit and a generalised fish-eye lens model.

A least squares fit was performed directly on a radius versus elevation plot, shown in Fig. 3. The fit is good with a $\chi^{2}$ value of $\sim 0.1$, and the complete fit equation is given by Eq. (2).

$$
\begin{aligned}
& r(\theta)=1.37 \times 10^{-5}+0.0034 \theta+0.0012 \theta^{2} \\
& -0.0022 \theta^{3}+0.0014 \theta^{4}+0.00036 \theta^{5}
\end{aligned}
$$

A direct comparison with Eq. (1) can be made by reexpressing it as Eq. (3), using a scale factor parameter $\alpha$, and a radial mapping parameter $\beta$.

$r=n \cdot f \cdot \alpha \sin (\beta \theta)$

where $n=8 / 18$, a reduction factor due to the other optics in the all-sky system. The radial distance from the optical axis on the CCD is matched against the radius calculated by the model; and best fit values of $\alpha$ and $\beta$ are shown in Table 1 . These values are compared with values obtained by Thoby (2006) for a similar Sigma $8 \mathrm{~mm}$ fish-eye lens, and the ideal parameters in the simple fish-eye equation. The differences between the best fit and the Thoby parameters are likely to be the result of the other optics in the SCANDI all-sky imaging system. The differences are very small, with a root
Table 1. A table comparing the best fit and expected results. The expected values come from Thoby (2006), where an experiment was performed on the same Sigma $8 \mathrm{~mm}$ fisheye lens as SCANDI. This can be further compared with the coefficients in Eq. (1), shown in the last column of the table.

\begin{tabular}{cccc}
\hline & $\begin{array}{c}\text { Best fit } \\
\text { (Eq. 3) }\end{array}$ & $\begin{array}{c}\text { Thoby } \\
\text { parameters }\end{array}$ & $\begin{array}{c}\text { Simple fish-eye lens } \\
\text { parameters (Eq. 1) }\end{array}$ \\
\hline$\alpha$ & 2.17 & 1.88 & 2 \\
$\beta$ & 0.48 & 0.54 & 0.5 \\
\hline
\end{tabular}

mean square value of only $0.25 \%$. Eventually the direct least squares fit was chosen as a more precise fit to model the fisheye lens projection in SCANDI, and an azimuth and elevation associated with each pixel and SCANDI zone were found for future references.

\subsubsection{Look-direction projection onto detector}

It is important for intercomparison with co-located instruments to project SCANDI's 25 zones onto a sky map as shown by Fig. 4a, which includes the geographic map underneath, and the numbering of each sector. An altitude of $250 \mathrm{~km}$ was chosen for the projection because it is the approximate altitude of the $630 \mathrm{~nm}$ emission peak. The appropriateness of the choice of this altitude can be cross-checked by comparing the structure of localised features such as enhancements of electron density and $630 \mathrm{~nm}$ intensity from other instruments with overlapping fields-of-view, such as the EISCAT radars, the Svalbard FPI and a Meridian Scanning Photometer. This study will be discussed in a later paper.

The equivalent look directions at $250 \mathrm{~km}$ are calculated by simple geometry (Eq. 4), ignoring the Earth's curvature, where $R_{250}$ is the radial distance from the SCANDI to the height of the emitting volume, $h$. The $250 \mathrm{~km}$ zone grid is then mapped using an azimuthal equidistant projection (IDL Online Help, 2006).

$R_{250}=h \tan \theta$

Different zone divisions were also tested. In the first winter of operation (December 2006-March 2007), the 25 zones were divided into approximately equal areas on the detector CCD chip, as shown by Fig. 4b, which also demonstrates a simulation of the Airy function image produced by the Fabry-Perot etalon. After they were projected onto $250 \mathrm{~km}$ altitude, zones from the outer SCANDI rings were seen to be elongated, with larger projected area than those of the inner rings as shown in Fig. 4a. Table 2 shows the inner and outer zenith angles for each ring for winter 2006-2007.

For data analysis purposes (i.e. equal weighting of all zones when taking averages or making comparisons) it was decided that it would be more convenient to split the 
Table 2. A table showing the inner and outer zenith angles for each ring, and the associated solid angles. For the first winter (2006-2007) the SCANDI zones were divided according to equal area on the CCD chip.

\begin{tabular}{|c|c|c|c|c|}
\hline & \multicolumn{4}{|c|}{ Season $2006 / 07$} \\
\hline & $\begin{array}{c}\text { Inner zenith } \\
\text { angle } \theta_{1} \text { (degrees) }\end{array}$ & $\begin{array}{c}\text { Outer zenith } \\
\text { angle } \theta_{2} \text { (degrees) }\end{array}$ & $\begin{array}{l}\text { Solid angle } \\
\text { (steradians) }\end{array}$ & $\begin{array}{l}\text { Projected area } \\
\qquad\left(\mathrm{km}^{2}\right)\end{array}$ \\
\hline Centre & 0 & 16 & 0.24 & 16100 \\
\hline Inner ring 1 & 17 & 32 & 0.18 & 15100 \\
\hline Middle ring 2 & 33 & 49 & 0.15 & 22900 \\
\hline Outer ring 3 & 50 & 67 & 0.14 & 69200 \\
\hline
\end{tabular}

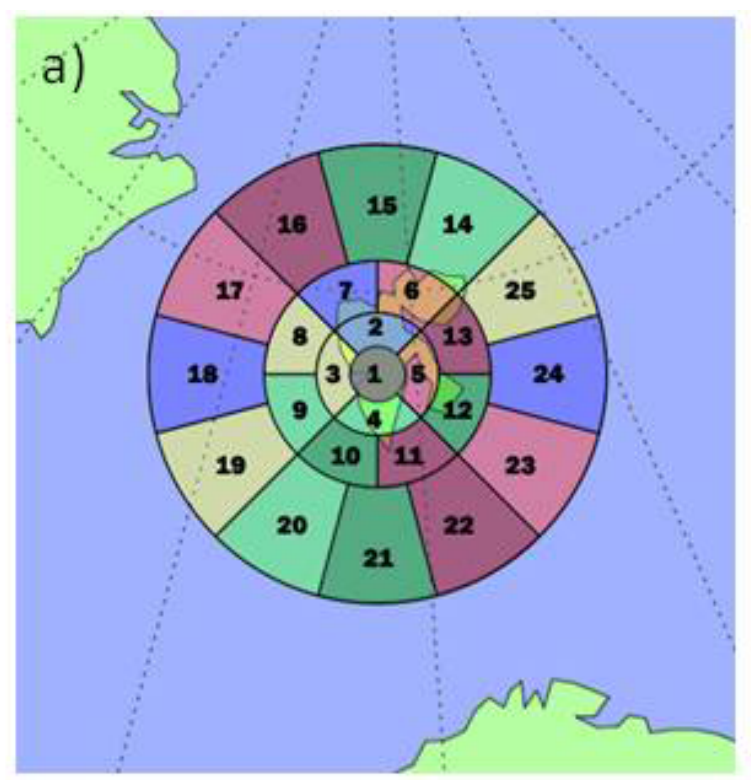

b)

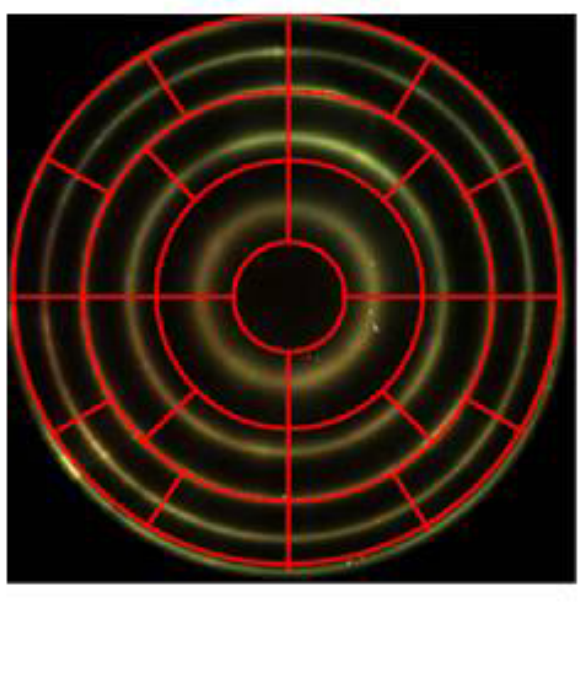

\section{C)}

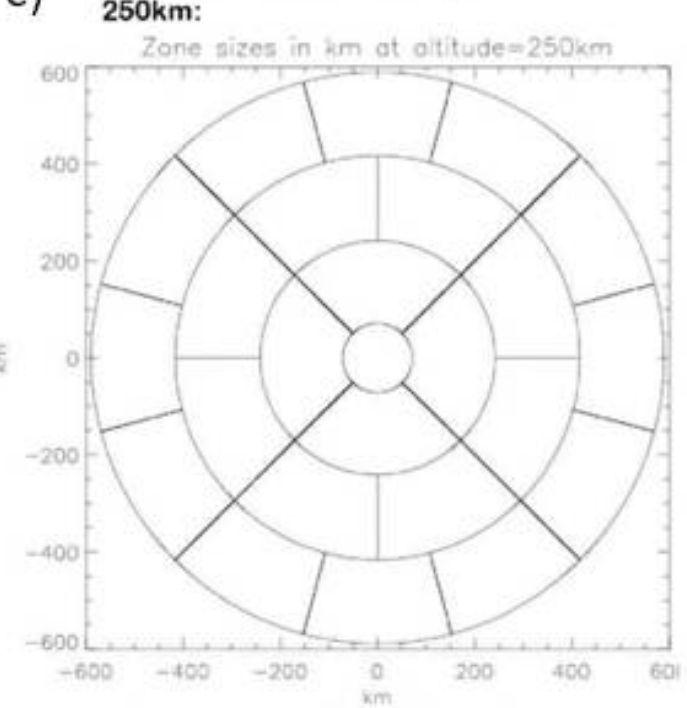

d) cco Chip:

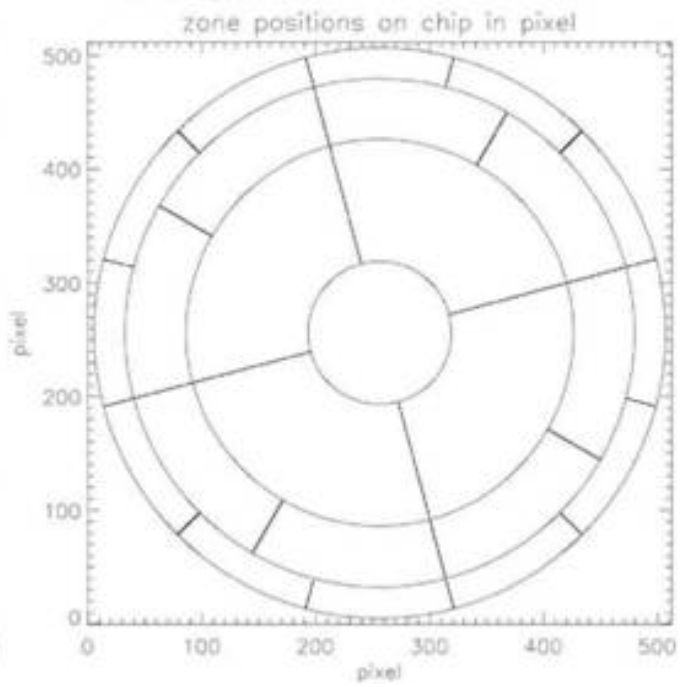

Fig. 4. Illustration of the SCANDI zones. For the first winter 2006-2007 (a) projection of the zones onto an altitude of $250 \mathrm{~km}$ when the zones were divided evenly on the CCD chip, as shown by (b). Here also is shown the Airy function image. From winter 2007-2008 onwards the zones were set to produce an equi-area projection on the sky (c), and (d) shows the projection on the CCD chip. 
Table 3. A table showing the inner and outer zenith angles for each ring, and the associated solid angles. From the second winter (2007-2008) onwards the SCANDI zones were divided according to roughly equal area projected on the sky, except for the central zone.

\begin{tabular}{ccccc}
\hline & Inner zenith & \multicolumn{2}{c}{ Season 2007/2008 onwards } & \\
Outer zenith & Solid angle & Projected area, \\
angle $\theta_{1}$ (degrees) & angle $\theta_{2}$ (degrees) & (steradians) & $\left(\mathrm{km}^{2}\right)$ \\
\hline Centre & 0 & 16 & 0.24 & 16100 \\
Inner ring 1 & 17 & 44 & 0.38 & 41700 \\
Middle ring 2 & 45 & 59 & 0.16 & 45100 \\
Outer ring 3 & 60 & 67 & 0.065 & 45500 \\
\hline
\end{tabular}

SCANDI field-of-view according to equal emission volume/solid angle. As a consequence, the zone areas decrease as the distance from the image centre increases. The equal solid-angle projection scheme is shown in Fig. 4c, which shows the projection on the sky, and Fig. 4d, the projection on the EMCCD chip. Table 3 shows the inner and outer zenith angles for each ring from winter 2007-2008 onwards. The derivation of the solid angle and the consequent area projected on the sky is shown in the Appendix.

The horizontal spatial resolution from a 25 -zone grid is variable in terms of the radial and azimuthal spatial resolution, but on average is about $200 \mathrm{~km}$, as indicated by Fig. 4a. Twenty five zones are suitable for low intensity emission as exists in this extended period of solar minimum. However, it is possible to increase the resolution for small-scale structure investigations: two new grids with 51 and 91 zones have also been constructed, with respective resolutions of $\sim 100 \mathrm{~km}$ and $\sim 50 \mathrm{~km}$. These grids and the associated phase map reductions can be implemented when the emission intensity becomes higher, as we progress into solar maximum. Indeed the latest University of Alaska SDI has 115 zones. However, it can be seen from Fig. 4d, which shows the projection on the chip, that for an equal solid-angle projection, the outer zones divisions on the CCD chip become very narrow and cover rather few pixels. This creates a problem that needs to be examined. It will be necessary to determine if the reduction in the number of pixels in the outer zone will mean fewer photons are detected, resulting in a poor signal to noise ratio which might make the data unusable. However, in theory at lower elevations the line-of-sight look direction passes through a greater depth of the emission layer, so more photons should be seen, but this is counteracted by increased atmospheric extinction.

\subsection{SCANDI data reduction}

\subsubsection{Wind and temperature determination}

As described in Sect. 2.2, each period of sky observation results in a profile of intensity versus scan step number (equivalent to wavelength) for each zone within the SCANDI sky map. For each of these profiles spectral width, peak wave- length, and source intensity along with an estimate of the background intensity are calculated by a nonlinear leastsquares curve-fitting procedure. For each observing zone, experimentally determined instrument functions are convolved with trial Gaussian emission functions. The goodness of fit of the transform of each trial to the transform of the data is estimated by calculating the chisqr residuals. The choice of parameters that gives the smallest chisqr is taken as the best fit and is used to compute the temperature, line-of-sight bulk velocity and intensity associated with the relevant region corresponding to the SCANDI zone. By combining the relevant results from each of the zones it is possible to produce maps of each parameter across the entire SCANDI field of view.

The instrument function for each zone is determined by using a stabilized He-Ne laser as the source for a scan similar to that implemented during sky observations. The profiles are adjusted to take into account the difference in wavelength between the laser $(632.8 \mathrm{~nm})$ and sky observations $(630.0 \mathrm{~nm})$. The laser scans are performed once each night and the calibration lamp observations $(630.48 \mathrm{~nm})$ are taken several times through each night of observations. The laser and calibration lamp together provide continuous monitoring of all of the factors that influence the stability and performance of the instrument.

\subsubsection{Software implementation}

Algorithms using the IDL development language based on Conde (private communication) have been developed to implement accurately and rapidly the profile fitting and atmospheric parameter determination described briefly in Sect. 2.4.1 and in more detail in Conde and Smith (1997). The basic fitting routines have been augmented to include the instrument functions and sky data output obtained by the control software. The routines were tested on FPI data and found to reproduce the results obtained using FPI programmes.

\subsubsection{Establishing a zero baseline}

In common with the treatment of FPI line-of-sight (LOS) winds, the SCANDI LOS winds need to be calculated by 
a) Total no. of dato points in each hour, 07/08 season

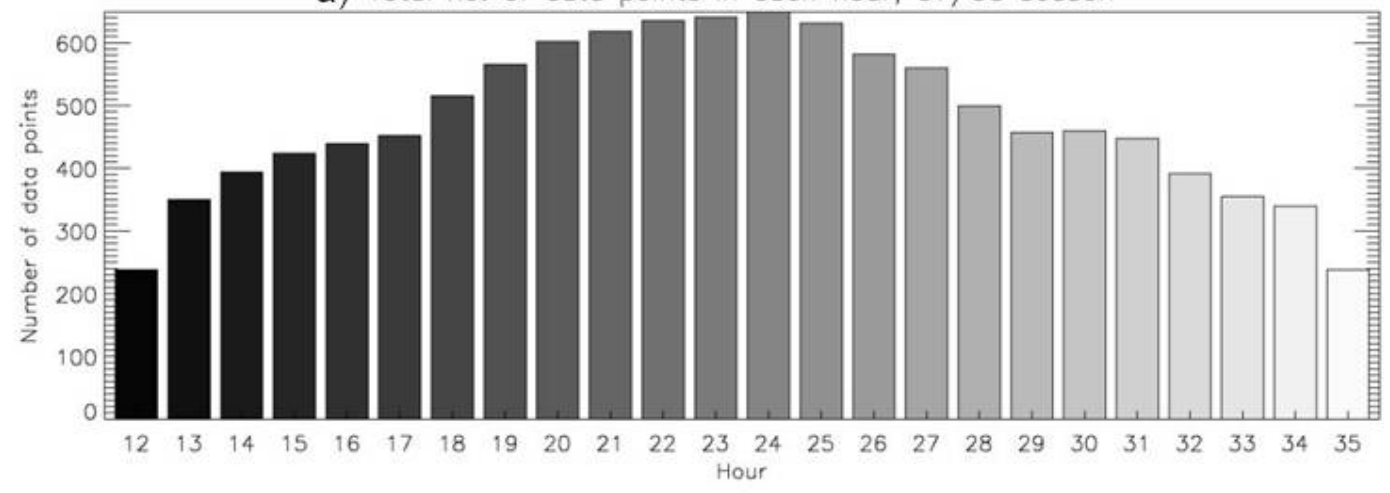

b) Total no. of data points in time zone, 07/08 season

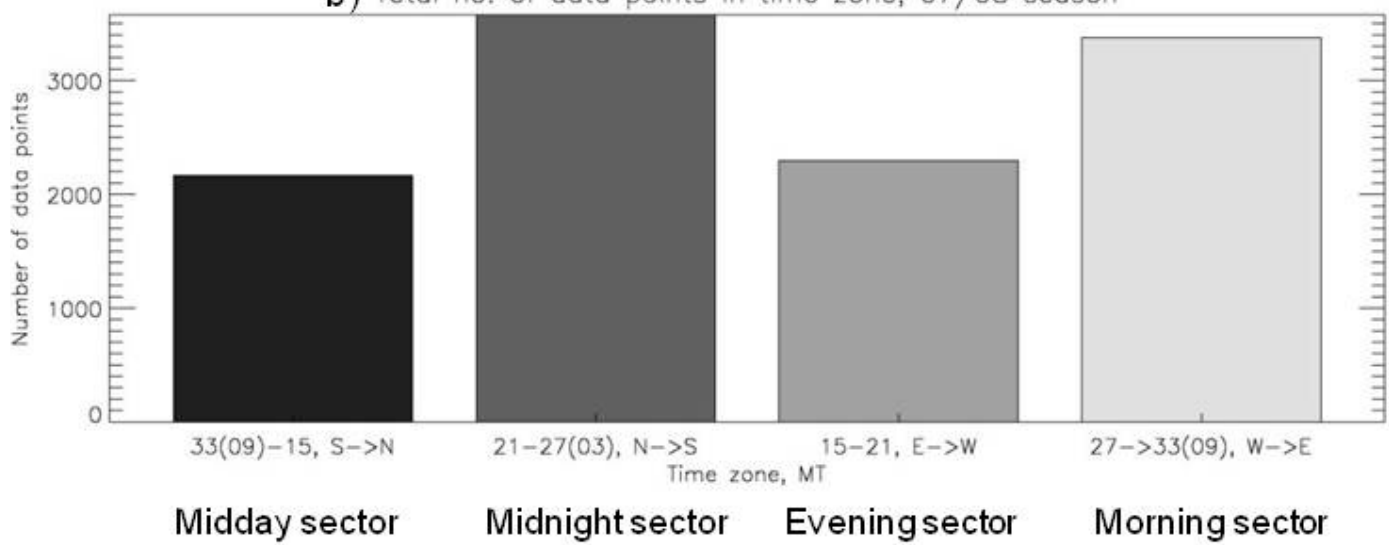

Fig. 5. Frequency distribution of data points during the winter of 2007-2008.

determining the deviation of the fringes from a zero-Doppler shift baseline, i.e. the position for the fringe peak in the absence of a wind. The standard FPI technique used to calculate the zero-shift position presumes that the average zenith wind during a night of observation is zero. The procedure is that the average offset between the calibration lamp peak and the zenith peak is calculated for an individual night. Then this offset is added to the 15 min interpolated calibration lamp peak data to produce a baseline. Establishing a reliable zero-Doppler shift baseline is a very important procedure in order to have confidence in the wind calculations. For SCANDI we have tested a number of zero baseline strategies over extended periods which are described below. The success of each baseline strategy is tested by comparing the independently calibrated FPI winds from each night with those calculated from SCANDI for the comparable zones.

In November 2007 the SCANDI and FPI instruments were relocated from the Adventdalen hut in the valley to the newly built Kjell Henriksen Observatory (KHO, $78.148^{\circ} \mathrm{N}$, $16.043^{\circ} \mathrm{E}$ ) on the mountain near the EISCAT Svalbard Radar (ESR). Figure 5a shows the frequency distribution of the number of SCANDI data points collected for each hour of UT time for the season December 2007-March 2008. Observations are made only when the zenith angle of the Sun is more than $6^{\circ}$ below the horizon. At this latitude a full $24 \mathrm{~h}$ of observation are possible during the months of December and January. Before and after this time the length of the period of observation rapidly builds up from September equinox and falls off towards March equinox. Figure $5 b$ shows the frequency distribution of the number of SCANDI data points for the four 6-h periods of Universal Time: midday (09:00-15:00 UT), midnight (21:00-03:00 UT), evening (15:00-21:00 UT) and morning (03:00-09:00 UT). The middle of the period of observation, local midnight, is near 23:00 UT, which means that the midday period is not as well sampled as midnight.

When considering a single all-sky measurement, it is possible to create a zero Doppler shift baseline by averaging the LOS winds from every sector within a single ring. This is because a ring-average of a uniform horizontal wind should give a value of zero (e.g. averaging LOS winds from the 2 nd ring: zones 5-13). This is illustrated by considering the unit vector $\underline{\hat{r}}(i, j)$ describing the look direction for the $i$-th zone in the $\bar{j}$-th ring. If the wind is given by $U$, then each zone sees a LOS wind component $U_{\text {los }}$ given by Eq. (5), and the average around a single ring gives $<U_{\mathrm{los}}>=0$.

$U_{\mathrm{los}}(i)=\underline{U} \cdot \underline{\hat{r}}(i, j)$ 
a) CAL overnight average (zone 2)

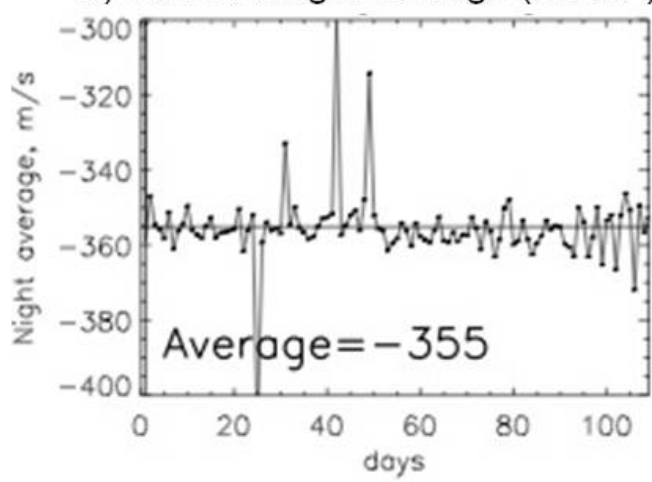

b) SKY overnight average (zone 2)

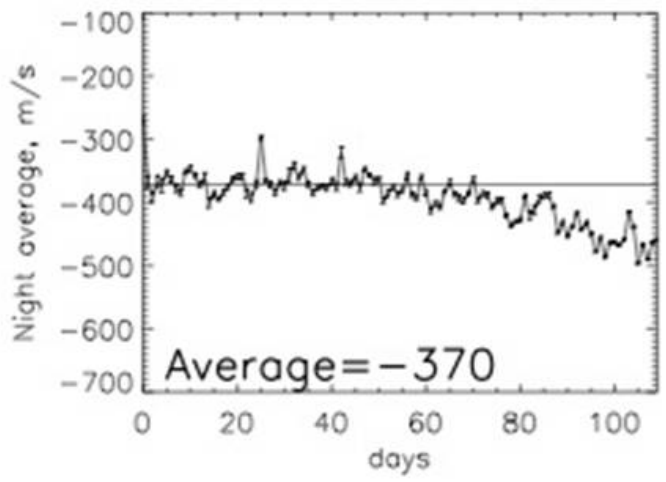

Fig. 6. Variation of the (a) calibration lamp peaks (CAL), and (b) $630 \mathrm{~nm}$ emission peaks (SKY) throughout the winter of $2007-2008$ for zone 2 .

However, the average position depends on the stability of the instrument, and to achieve this the etalon is kept in a sealed temperature controlled housing, and the SCANDI is kept in its own room at the $\mathrm{KHO}$ where there is minimal disturbance. Figure 6a shows the stability of SCANDI during the period December 2007-February 2008 by plotting the calibration lamp (CAL) data for each of the 25 SCANDI zones. Four calibration measurements are made each day. The nighttime average value of the line-of-sight peak position shifts have been converted to equivalent LOS wind speeds using an arbitrary baseline. There is a small variation about a mean value for each zone, with a few outliers when the calibration lamp fails to light immediately, which indicates good stability over the 122 day period of observation (1 December 2007-31 March 2008).

In contrast, Fig. $6 \mathrm{~b}$ shows that the nighttime mean LOS wind speeds from the $630 \mathrm{~nm}$ emission (SKY) are steady for the first 60 days and then the values start to drift away either upwards or downwards depending on the location of the zone. The reason for this drift is that the SKY data shows real wind speeds from Doppler shifts observed in the $630 \mathrm{~nm}$ emission. The first 60 days cover December-January, which is a period of $24 \mathrm{~h}$ darkness. After this time, the length of the observing period each night rapidly decreases as equinox approaches. Over a $24 \mathrm{~h}$ period the winds observed should tend to an average LOS wind value of zero owing to conservation of mass considerations applied to the global circulation of the atmosphere. The drift in the nighttime average wind value that is seen after the end of January occurs because the observing period shortens and the nighttime sample of winds is dominated by the net anti-sunward wind typical of the midnight sector.

The first 60 days' nighttime mean LOS wind speeds from the SKY measurements and the equivalent winds from the CAL measurements are found to be different for each zone, as shown in Fig. 7. The triangle points are SKY average data and the + signs are CAL average data. The $\mathrm{x}$-axis shows the zone number, from 1-25 (see also Fig. 4a). The vertical lines between zones 1-2, 5-6 and 13-14 delineate the inner ring, 1 st, 2 nd and 3 rd zone rings. These are absolute values. There is no baseline subtraction so the values range between -270 to $-500 \mathrm{~m} / \mathrm{s}$, rather than being centred on zero.

The dashed horizontal lines are the average values around each individual ring for the SKY data and the dot-dash lines are for the CAL data. These average values are considerably different when comparing SKY and CAL averages. The CAL average for ring 1 is around $15 \mathrm{~m} / \mathrm{s}$ less negative than the SKY, but about $30 \mathrm{~m} / \mathrm{s}$ more negative for ring 2 and $40 \mathrm{~m} / \mathrm{s}$ less negative for ring 3 . Thus there is no consistent trend between the CAL and SKY data. The 3 rings give different CAL averages, though within $10 \mathrm{~m} / \mathrm{s}$. The maximum difference between the SKY averages is $60 \mathrm{~m} / \mathrm{s}$ between the ring 2 and 3 average values.

The CAL average values show a small variation about the mean value for each ring. There appears to be a sinusoidal variation with underlying positive gradient. One and a half periods of the sinusoid are displayed for each ring. The amplitude of the sinusoidal variation increases from ring 1 through to ring 3 . The maximum amplitude is $20 \mathrm{~m} / \mathrm{s}$. Meanwhile the SKY average values show one complete sinusoidal period, with amplitude increasing from ring $1(\sim 10 \mathrm{~m} / \mathrm{s})$ to ring $3(115 \mathrm{~m} / \mathrm{s})$. This sinusoidal variation is caused by the etalons not being perfectly parallel. The different wavelengths of the CAL and SKY emissions account for the different trends. Consequently there is a systematic shift in the baseline for each sector that must be removed. The standard deviation of the average SKY LOS wind (around $\pm 50 \mathrm{~m} / \mathrm{s}$ ) for each zone is caused by the variability of the winds.

Figure 8 shows comparisons of the SCANDI ring 3 LOS winds when compared with the FPI winds for the night of 7-8 December 2007. The look directions of the ring 3 North, East, South and West sectors correspond to the FPI look directions, which are at an elevation angle of $30^{\circ}$. The large outliers in the SCANDI data occur when the intensities are 


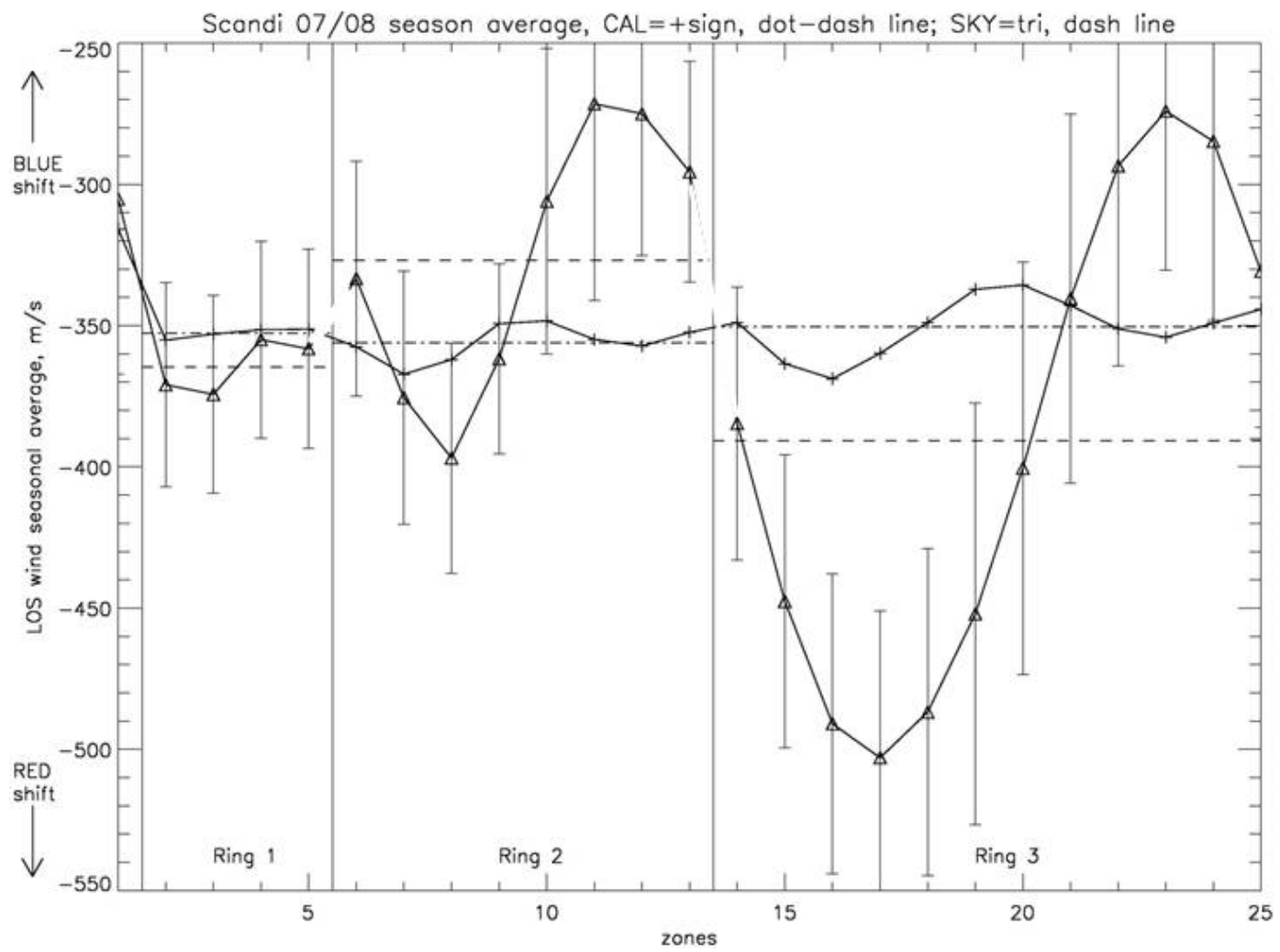

Fig. 7. Average position (December 2007-January 2008) of the CAL (stars) and SKY (triangles) peaks for each zone shows a sinusoidal variation around each ring. The peak positions are offset from an arbitrary baseline. The horizontal lines indicate the average position for each ring: $\mathrm{CAL}=$ dot-dash; $\mathrm{SKY}=$ dashed. The vertical lines indicate the standard deviation of the data. The small standard deviation of the CAL data indicates good thermal stability for SCANDI, while that of the SKY data indicates the natural variability of thermospheric winds.

North
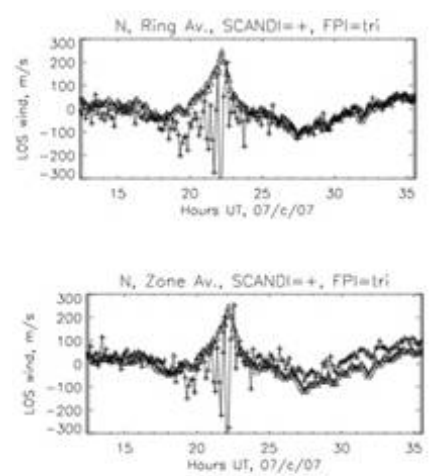

East
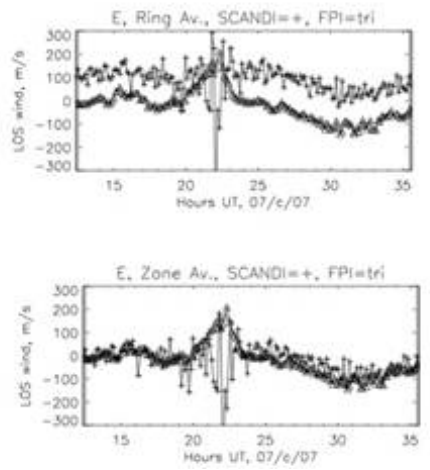

South
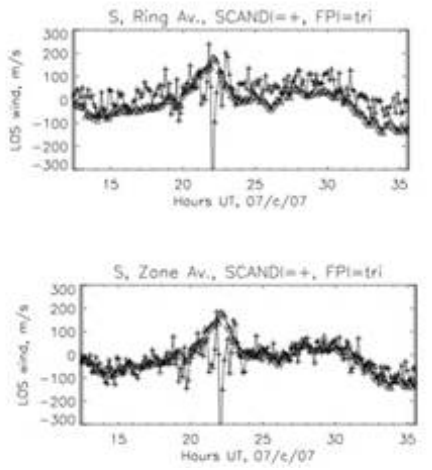

West
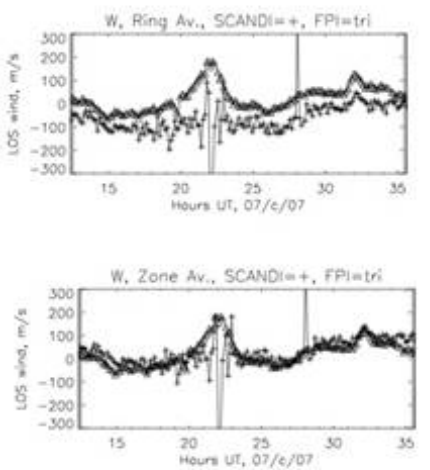

Fig. 8. Comparison of SCANDI LOS winds with FPI LOS winds using two methods of determining the zero-Doppler shift baseline. In the top row the baseline for each zone is determined from the average offset for each zone, i.e. each data point (triangle) in Fig. 7. There is good agreement between the SCANDI and FPI LOS winds. In the bottom row the baseline for all zones in a given ring is determined from the average offset for that ring, i.e. the dashed lines in Fig. 7. There is a large systematic offset between the SCANDI and FPI LOS winds.

very low. Although the FPI has only a $1^{\circ} \mathrm{FOV}$, the whole of the etalon area gathers the photons, thus giving higher signals than the SCANDI during periods of low intensities. The SCANDI winds in the top row are derived by using the same ring-averaged baseline value for every zone within a ring. The winds in the bottom row are derived by using an individual zone-average baseline calculated for that zone. Clearly there is a better match between SCANDI and FPI winds 


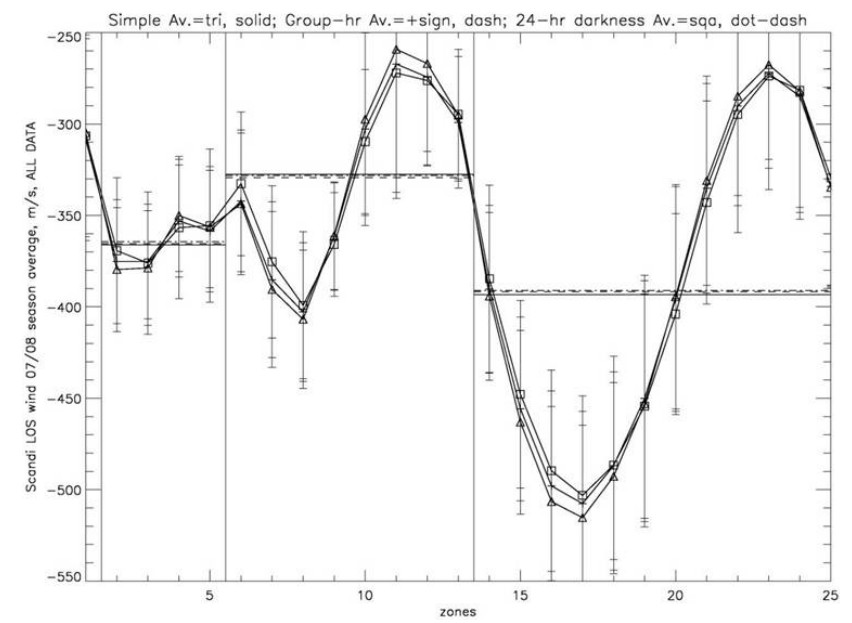

Fig. 9. Same as Fig. 7, but showing only SKY data. Here the data are averaged in 3 different ways (see text).

shown by the bottom row of Fig. 8, compared with the top row. In the top row can be seen a large systematic difference between the SCANDI and FPI winds, for example subtracting SCANDI winds from FPI winds gives a large positive value $(\sim 100 \mathrm{~m} / \mathrm{s})$ for the East look direction, and a slightly smaller negative value for the West look direction.

Figure 9 shows the average LOS winds for each zone for 3 averaging methods. The triangles show the simple average for each zone over all the data points collected for this season, where the horizontal solid line represents the average around the sectors within each ring, i.e. the ring-average. The + symbols show the average values where first the average for each hour is determined, and then the average of $24 \mathrm{~h}$ is calculated. The dashed lines show the ring-averages for each ring. The squares show the averages using only the December-January data, with the ring-averages shown by the dot-dash lines.

There is a small difference of less than $10 \mathrm{~m} / \mathrm{s}$ in the 3 sets of averages which is caused by the different weighting of the data according to the size of the samples used. Using all the data points, as in the 1st case, biases the averages to those times and dates with the largest number of observations. The 2 nd and 3 rd cases give a uniform weight to each hour, but there will be a seasonal influence since the 2nd case includes both solstice and equinox months, while the 3 rd case includes only solstice months when there are a full $24 \mathrm{~h}$ of darkness every night. The $3 \mathrm{rd}$ case gives the best values for the zero Doppler shift baseline. However, the 3 ring-averages for each ring are very close in value, within $\pm 1 \mathrm{~m} / \mathrm{s}$, and the seasonal difference between the individual zone averages for the 2 nd and $3 \mathrm{rd}$ sets is less than $5 \mathrm{~m} / \mathrm{s}$, well within the $\pm 50 \mathrm{~m} / \mathrm{s}$ standard deviation of the average winds and within the error for fitting the peak.

Figure 10 shows the SCANDI-FPI LOS winds Pearson correlation coefficients for the 4 primary look directions. The
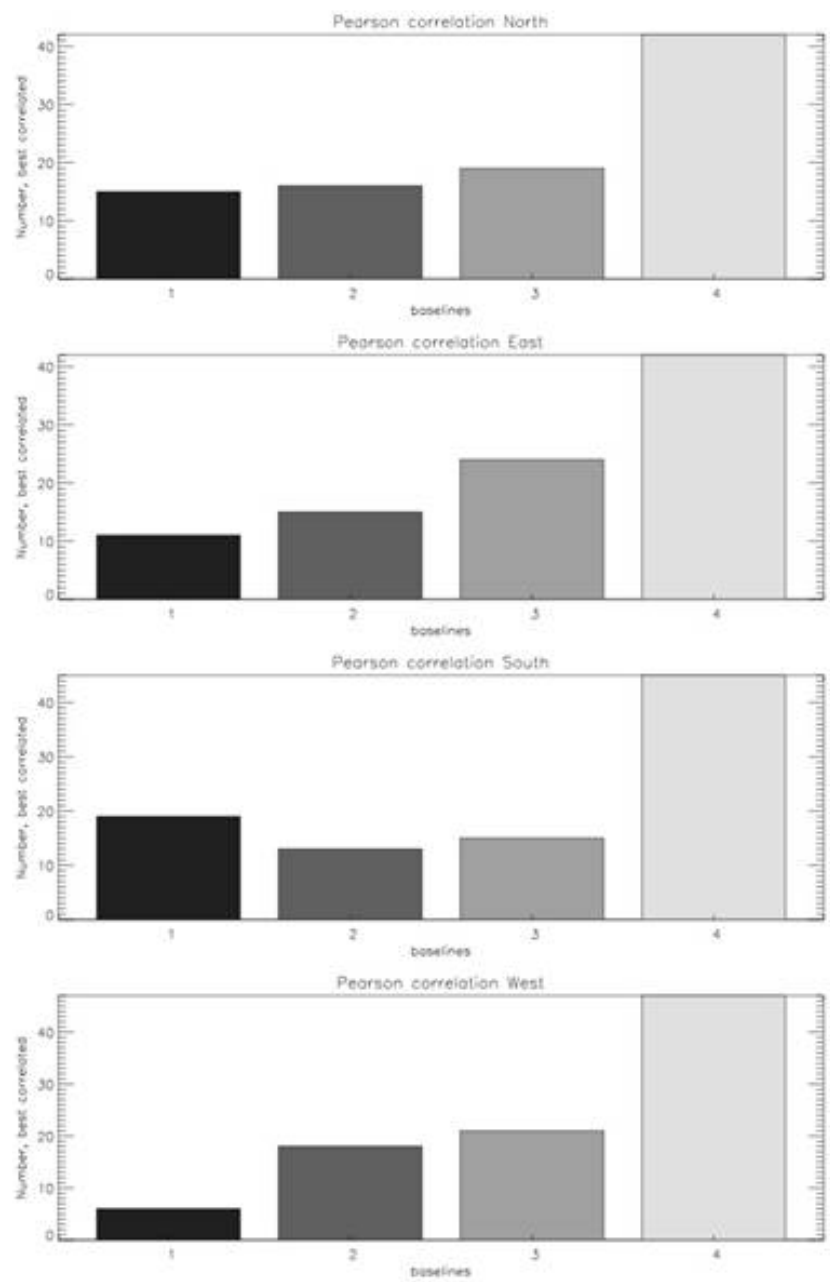

Fig. 10. Pearson cross-correlation of SCANDI and FPI LOS winds where 4 different baselines are used to determine the SCANDI LOS winds (see text).

SCANDI winds are calculated using 4 different baselines: an average baseline calculated using the night of observation; an average from the week; an average from the month and finally an average using the whole winter of observations. For all look directions the best agreement between the FPI and SCANDI data comes from using the whole winter. It is interesting that for the meridional look directions there is little difference in using an average baseline from a single night or a month, while for the zonal directions there is a clear trend of improvement in agreement when a longer period of data is used for the average. This would imply that the average baseline calculated from a whole winter of observations is optimum.

The process of determining the baseline, illustrated by Figs. 5-10, serves as a warning for the need for considered sampling. Our conclusion is that it is necessary to average all of the samples from within each zone during December to January (when there is continuous darkness) to establish the 

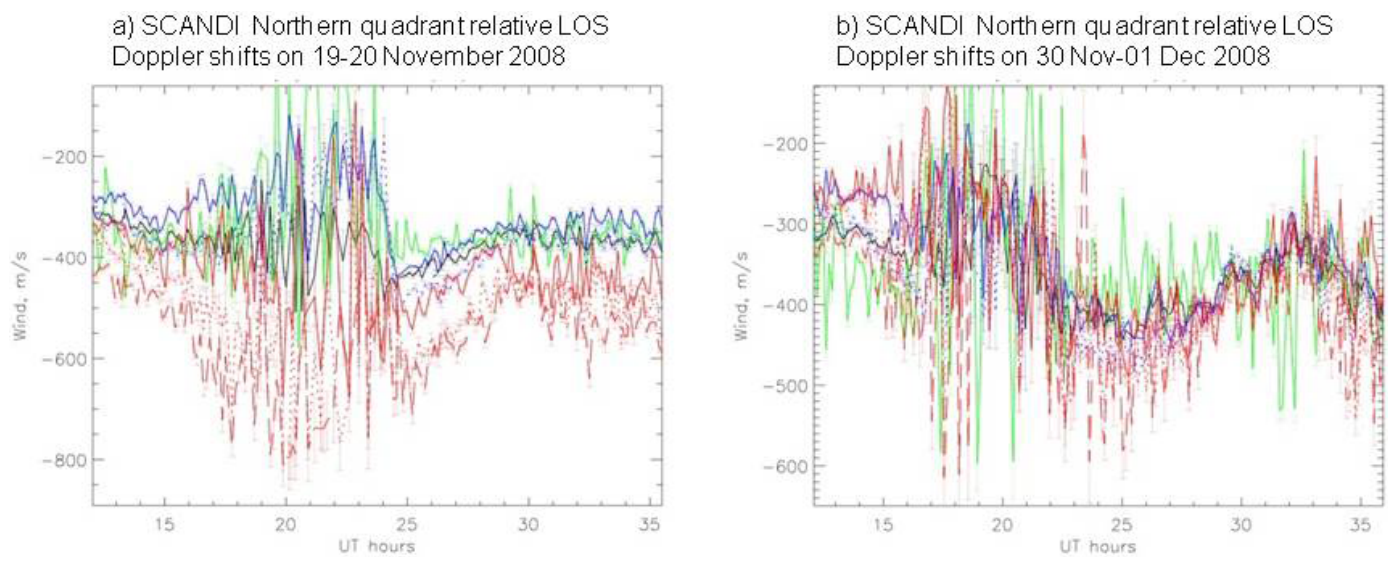

Fig. 11. The effect of fine-tuning the parallelism of the etalon is to make the baselines of all the rings similar. (a) SCANDI LOS winds (without baseline correction) seen in the quadrant to the North on the night of the 19 November 2008 before the etalon parallelism was fine-tuned. There is a large systematic difference between rings. (b) SCANDI LOS winds (without baseline correction) seen to the North on the night of the 30 November 2008 after the etalon parallelism was fine-tuned. The systematic difference between rings is small. Zone 1 $($ inner circle $)=$ green; zones $2-5($ ring 1$)=$ black; zones $6-13($ ring 2$)=$ blue; zones $14-25($ ring 3$)=$ red.

best zero baseline for that zone. This accounts for systematic instrumental error better than by taking a baseline from a ring average from a single night. The latter method is suitable for quick analysis of the data as they are being collected, but is not the best method because it relies on the assumption that the net LOS wind around a ring is zero over the period of observation. When the period of observation is significantly less than $24 \mathrm{~h}$, i.e., any night except for December-January, then this assumption is invalid.

\subsubsection{Fine-tuning the etalon parallelism}

With the simple FPI the Airy function around the whole $1^{\circ}$ field-of-view is reduced to a single radius-squared profile. The instrument function includes the effects of broadening of the fringes due to poor centring of the image, vignetting due to finite aperture size and any slight deviation from parallel etalon surfaces. Critically, the FPI instrument function is the same for all look directions since the direction of view is determined by the pointing direction of the mirror.

The look directions of the SCANDI instrument are determined by the location of each zone, as described in the Sky Mapping Sect. 2.3. There are a fixed number of steps made by the piezo-electric spacers. The scaling factor for the conversion from geometric step length to wavelength is achieved by calculating how many steps make a free spectral range; in other words what number of steps are required so that the $n$-th peak moves to the position originally occupied by the $(n+1)$ th peak. The effect of the etalons being off-parallel should not have an important effect on the calibration of the SCANDI data, as long as the degree of parallelism at the beginning is maintained throughout the scan so that the instrument function is the same for all pixels. In order to achieve this, the three offset signals applied to the capacitance bridge circuits must track perfectly with each other. The electronic gain of each offset channel is has to be very carefully adjusted to ensure parallelism at each end of the scan. This can be verified by the superposition of the intensity versus radius-squared plots obtained at each end of the scan.

During the processing of data from the period OctoberNovember 2008, it was realized that the etalon was very slightly off parallel. On the 26 November 2008 the APL team returned to Svalbard and fine-tuned the etalon parallelism, which improved the consistency of the calculated LOS winds. Figure 11a shows pure LOS winds seen in the north quadrant, with no baseline correction, calculated for the night of 19-20 November 2008, before the etalon was fine-tuned and Fig. 11b shows LOS winds after tuning, on the night of 30 November-1 December 2008. Each contains data from all the zones within the north quadrant. The green line in Fig. 11a is the central zone; the black lines are zones in the inner ring; the blue lines are ring 2 zones and the red lines represent zones in the outermost ring 3 .

Before the etalon was tuned (Fig. 11a) there is a large spread of values in the pure LOS winds measured in the different rings. The outermost ring 3 LOS winds in the north are systematically displaced by about $-150 \mathrm{~m} / \mathrm{s}$ from the inner rings' LOS winds. The ring 1 and 2 LOS winds in the east and south quadrants (which are not shown) are systematically displaced from each other by about $100 \mathrm{~m} / \mathrm{s}$. The differences are systematic and more than what is expected from taking into account the different azimuths and elevations represented by each zone and consequent mixing of meridional, zonal and vertical wind components. After tuning, the LOS winds from all rings are more reasonably consistent with each other (Fig. 11b).

Fine-tuning the etalon also results in a clear reduction in the systematic differences in the neutral temperatures 


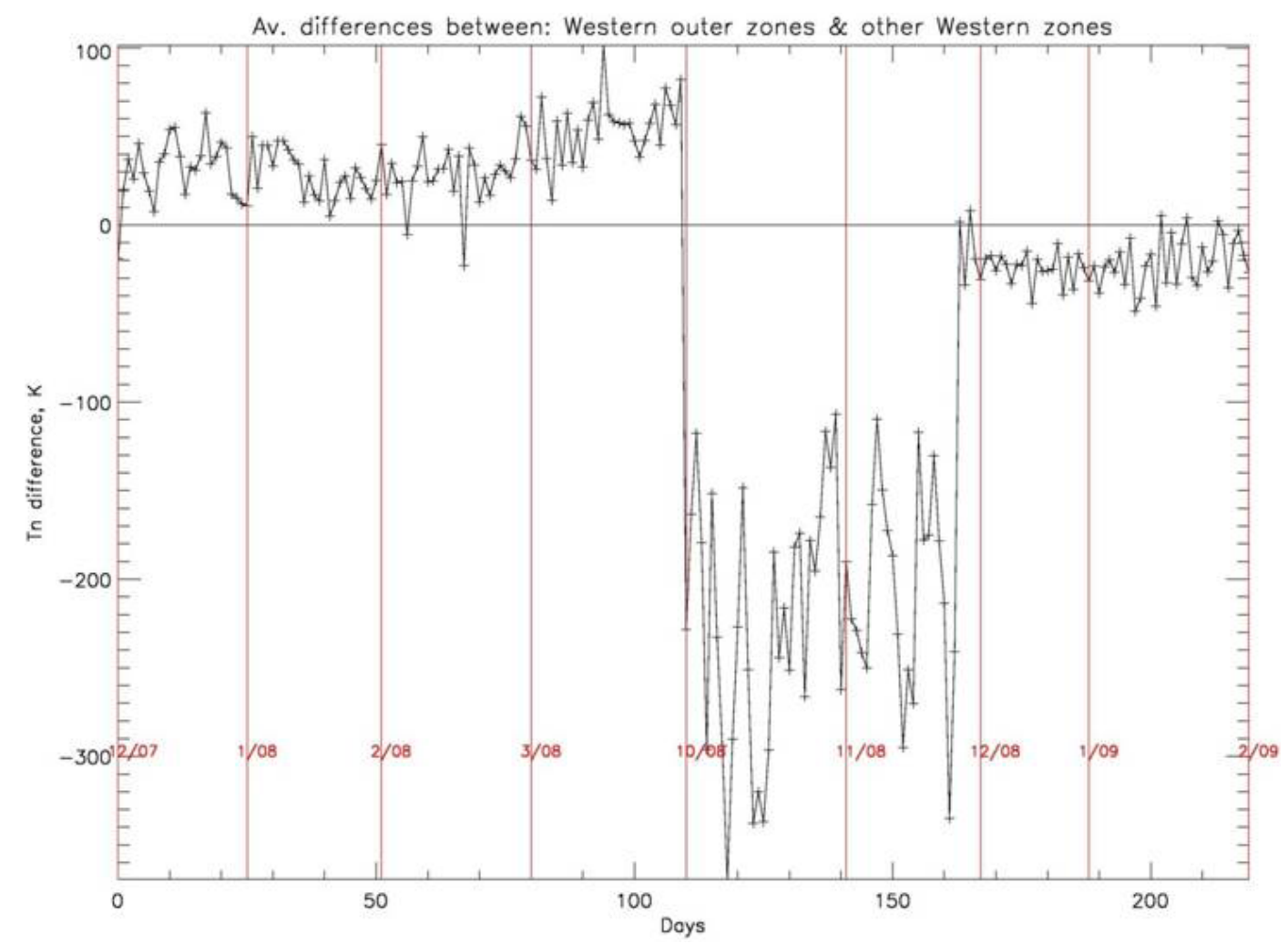

Fig. 12. Average offset of temperatures in ring 3 compared with temperatures in the inner rings for the West quarter zones.

calculated from each of the 3 rings. Figure 12 shows the average difference between the temperatures in ring 3 compared with those in the inner rings of the west quadrant. During the period October-November 2008 when the etalons were slightly off-parallel, there was a large systematic offset of around $200 \mathrm{~K}$. In the previous winter's observations, and after tuning the etalon at the end of November 2008, the offset was only a few 10 s Kelvin. All these systematic offsets must be removed when determining the true temperature.

\section{Initial observations}

\subsection{SCANDI in fixed etalon gap mode and very high spatial resolution}

The most important strength of SCANDI is to allow investigation of meso-scale behaviour. In the first month after installation we tested the instrument with a fixed gap mode as used by the first generation of Doppler imagers. The data quantity for the fixed gap mode was small enough to allow us to store the full 2-D image using a series of integration times, which varied from 60-300 s. Three rings were imaged and this provided the opportunity to investigate the centring of the image and test out different spatial resolutions. The limitation of the fixed etalon gap mode is that only the regions of sky that are projected onto these three rings can be measured. With the scanning mode, every single pixel is scanned through a full free spectral range thus imaging the whole sky. However, the huge data quantities involved with the scanning mode means that the raw data cannot be stored, only the fitted data as described in Conde and Smith (1997), which means that we cannot experiment with spatial scale after the data are collected. Comparison of the SCANDI measurements at different spatial scales with the FPI produced very interesting results.

The outermost ring has an average zenith angle of $57^{\circ}$, making it the closest to the FPI look direction, which is $60^{\circ}$. Figure 13 shows a comparison of SCANDI and FPI temperatures for the night of 23-24 December 2006 when SCANDI ring 3 data were integrated over a $90^{\circ}$ azimuthal range $\left(-45^{\circ}<\right.$ azimuth $<+45^{\circ}$; Fig. $\left.13 \mathrm{a}\right)$ and $10^{\circ}$ azimuthal range $\left(-5^{\circ}<\right.$ azimuth $<+5^{\circ}$; Fig. $\left.13 \mathrm{~b}\right)$. The red line shows the temperatures from the middle ring 2 and the blue line shows the outermost ring 3 . Both lines represent measurements towards the north that are separated by a radial distance of nearly $190 \mathrm{~km}$. The ring 3 data represent temperatures at a radial distance of $382 \mathrm{~km}$ to the north of Longyearbyen. Although the zenith angles in Table 2 indicate that the radial distance sampled by the zones in ring 3 while in scanning mode is $291 \mathrm{~km}$ (between $298-589 \mathrm{~km}$ ), the radial distance sampled by the peak of the Airy function in the fixed gap mode is only $100 \mathrm{~km}$ (between $332-433 \mathrm{~km}$ for the 

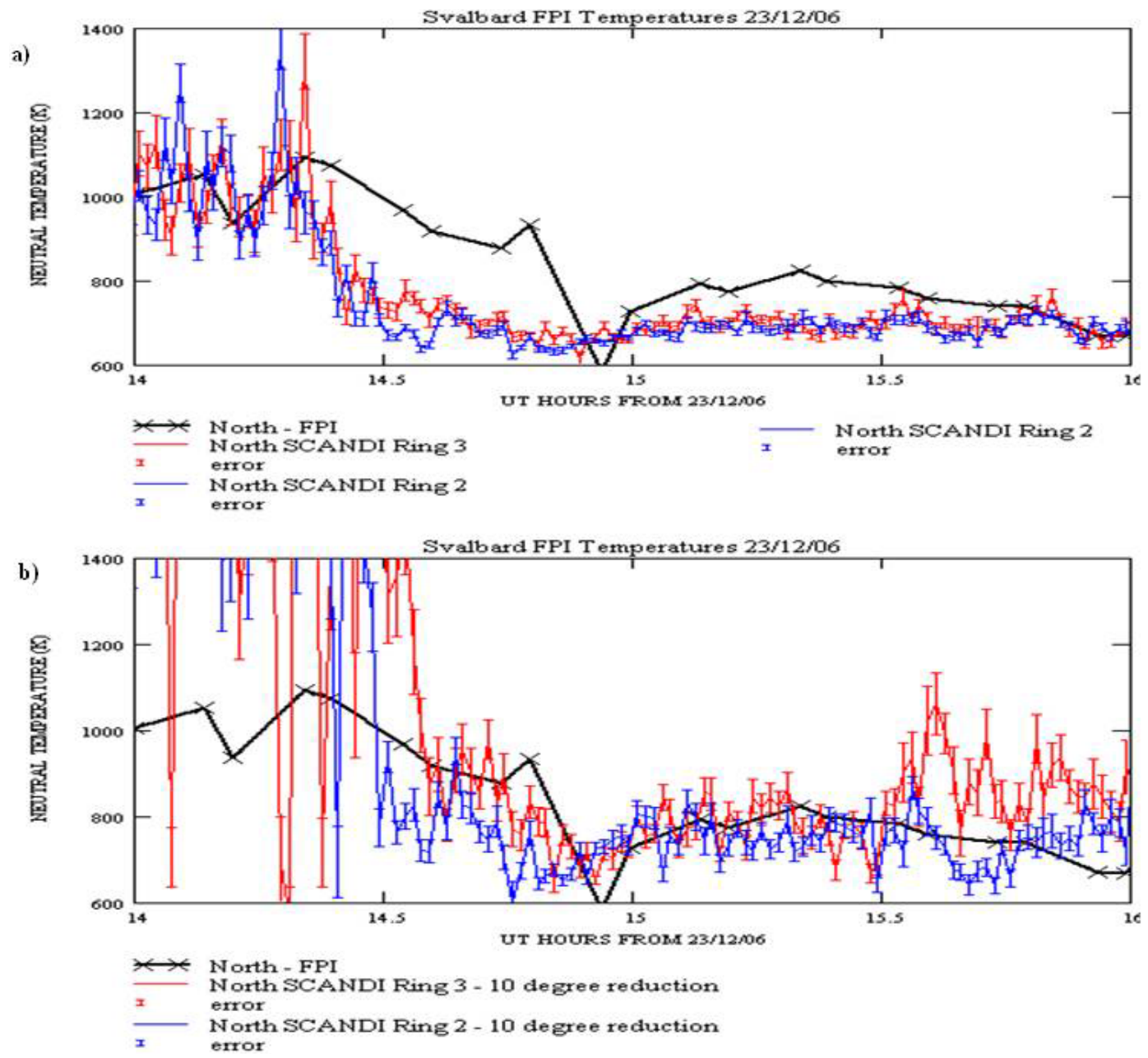

Fig. 13. Spatial structure around a scale-size of $100 \mathrm{~km}$ is demonstrated by comparing $T_{n}$ averaged over $\sim 900 \mathrm{~km}\left(90^{\circ}\right.$ azimuthal range) (Fig. 13a) with $\sim 100 \mathrm{~km}\left(10^{\circ}\right.$ range) (Fig. 13b). The black line shows the corresponding FPI measurement of $T_{n}$. Averaging the SCANDI $T_{n}$ over a smaller spatial range appropriate to the FPI look direction brings the two temperatures into agreement between 14.5-15.5 UT. A significant temperature gradient is seen after $15.5 \mathrm{UT}$ between rings 2 and 3, which represents a radial distance of $\sim 300 \mathrm{~km}$ from the centre of the SCANDI fov.

region between the full width half maximum intensity of the ring 3 peak). The maximum horizontal arc length covered by ring 3 over the $90^{\circ}$ azimuth range is around $900 \mathrm{~km}$, while the $10^{\circ}$ range is around $100 \mathrm{~km}$. For comparison the black line shows the corresponding FPI temperatures to the north using a $1^{\circ} \mathrm{FOV}$.

Figure 13 focuses on the period 14:00-16:00 UT which was a period of increased $630 \mathrm{~nm}$ intensity due to particle precipitation. The FPI temperature was high $(\sim 1000 \mathrm{~K})$ between 14:00-14:30 UT and then dropped to less than $800 \mathrm{~K}$ by 14:50 UT. This may seem counter-intuitive in the sense that this more active period may be expected to produce an increase in temperature. However, in this particular case, the likely scenario is that the average altitude for the $630 \mathrm{~nm}$ emission has dropped as a result of the precipitation because the auroral contribution to excitation dominates the airglow contribution. As a consequence the temperatures were characteristic of a lower altitude and hence lower temperatures were measured during the precipitation. This has been proposed as the reason for anomalous low temperatures measured using the $557.7 \mathrm{~nm}$ emission by Holmes et al. (2005) and for $630 \mathrm{~nm}$ by Griffin et al. (2008). On comparing the SCANDI temperatures with the FPI, Fig. 13a shows that when SCANDI is divided into a $90^{\circ}$ azimuthal range sector, the SCANDI temperatures both severely underestimate the neutral temperature measured by the FPI by $100-200 \mathrm{~K}$ due to integrating data over such a large area, and also give the impression of a very uniform temperature over the horizontal distance sampled by rings 2 and 3. However, when the data are integrated over a $10^{\circ}$ azimuthal range (Fig. 13b) the SCANDI temperatures become larger and agree with the FPI temperatures. Furthermore, large temperature gradients 


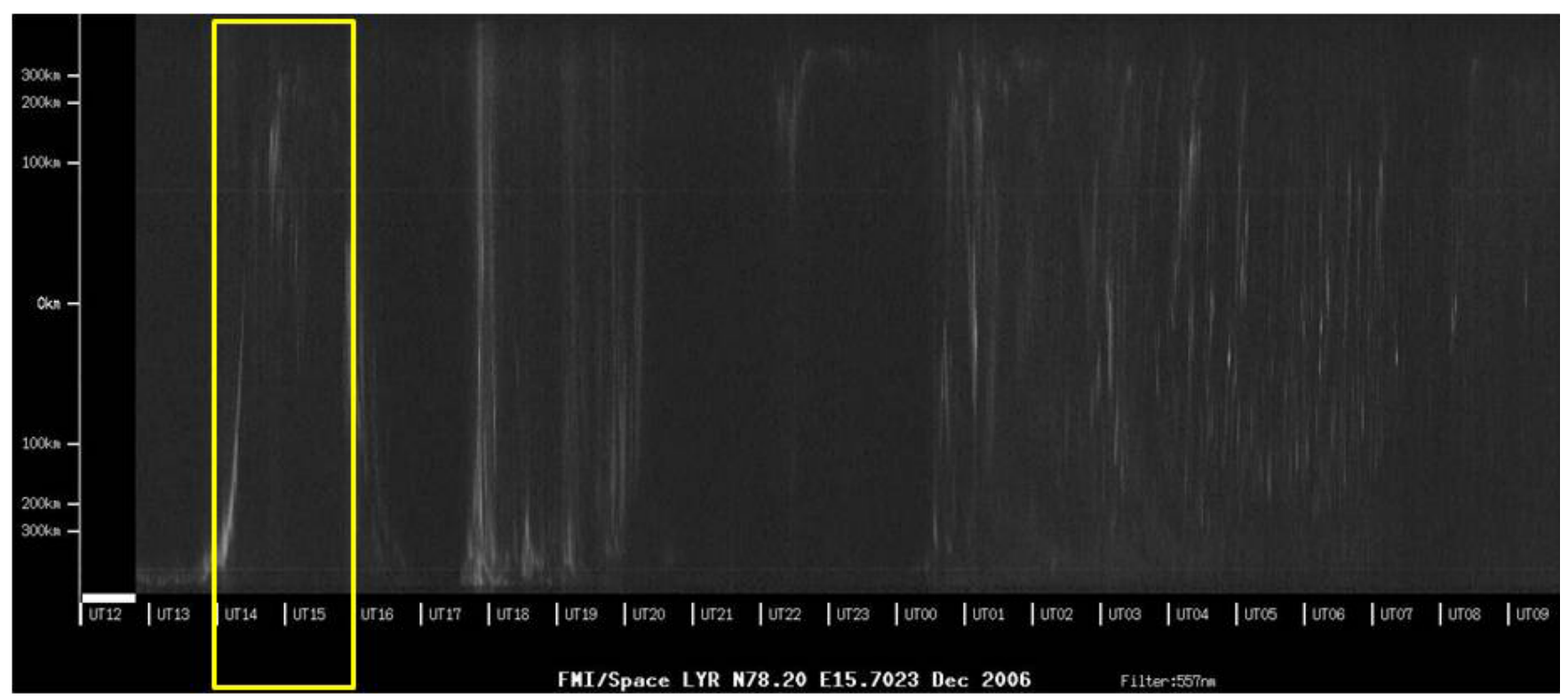

Fig. 14. FMI keogram for the night of 23 December 2006 showing how the aurora moves from south of Svalbard to the north during the period 14:00-15:00 UT corresponding to the change in the success of the match between SCANDI and FPI measurements of $T_{n}$ due to the signal to noise ratio.

appear between 15:30-16:00 UT: up to $200 \mathrm{~K}$ difference between rings 2 and 3 over this radial distance of $320 \mathrm{~km}$.

The FMI keogram using $557.7 \mathrm{~nm}$ filter for this night (Fig. 14) shows auroral activity moving from the south at 14:00 UT to the north of Longyearbyen by 15:00 UT before drifting back south by 16:30 UT. This is consistent with the spatial distribution of the $630 \mathrm{~nm}$ intensities seen over the SCANDI FOV. In the period 14:00-14.5 UT it can be seen that there is a good match with the FPI neutral temperature for the $90^{\circ}$ sector and bad for the $10^{\circ}$ sector. During this period the $630 \mathrm{~nm}$ intensities were very low in the north which means that the signal to noise ratio was poor for the $10^{\circ}$ sector and the temperature fitting routine seriously overestimates $T_{n}$. In contrast, the $90^{\circ}$ sector, which had accumulated more photons during the integration period, provides a better measure of the temperature when compared with the FPI $T_{n}$. Interestingly this may also indicate that there is a more uniform temperature field over large areas during geomagnetically quiet periods, with a scale size of several hundred kilometres, while in more active periods the thermospheric scale size drops to a hundred kilometres.

\subsection{SCANDI in scanning mode, and comparison with FPI}

The first observations of SCANDI using the scanning mode were compared with the Longyearbyen FPI in order to confirm the analysis process. Now the SCANDI FOV is divided into 25 zones. The FPI uses an elevation angle of $30^{\circ}$, which places the observed volume in the region of the outermost ring of zones, i.e., ring 3 . Figure 15 compares the SCANDI

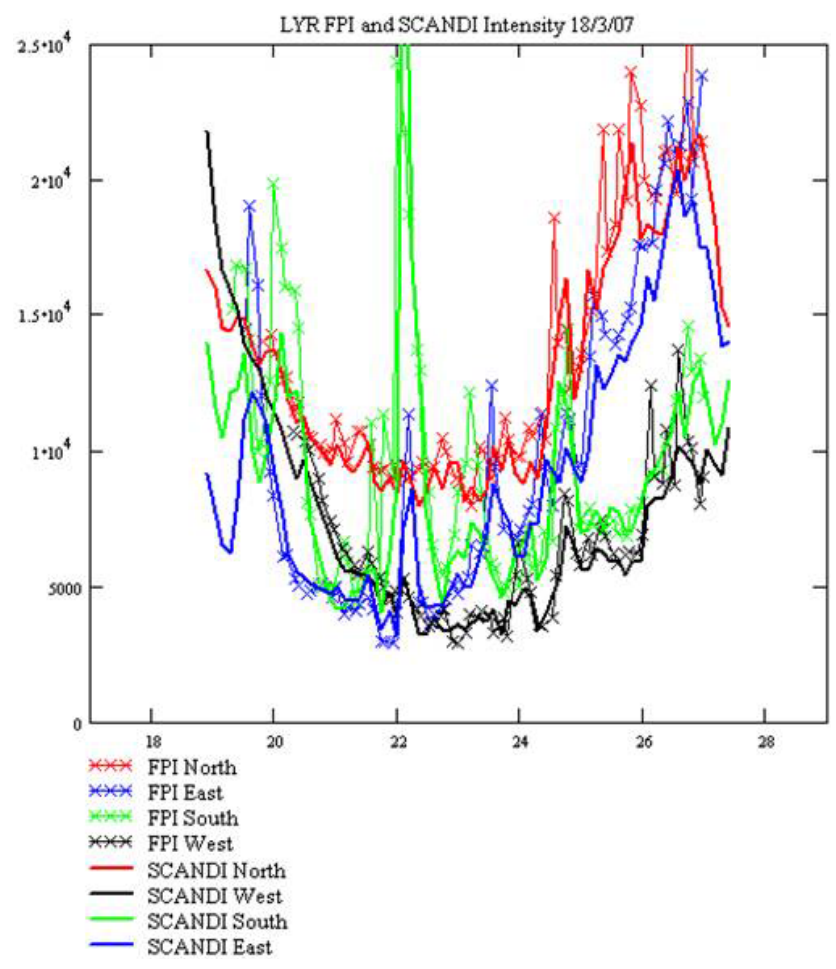

Fig. 15. SCANDI and FPI observations showing meso-scale structure in $630 \mathrm{~nm}$ intensity on 18 March 2007 over a field-of-view with radius $\sim 500 \mathrm{~km}$.

ring 3 intensities (lines) with the FPI intensities (lines with crosses) seen on the night of 18-19 March 2007 for the look 


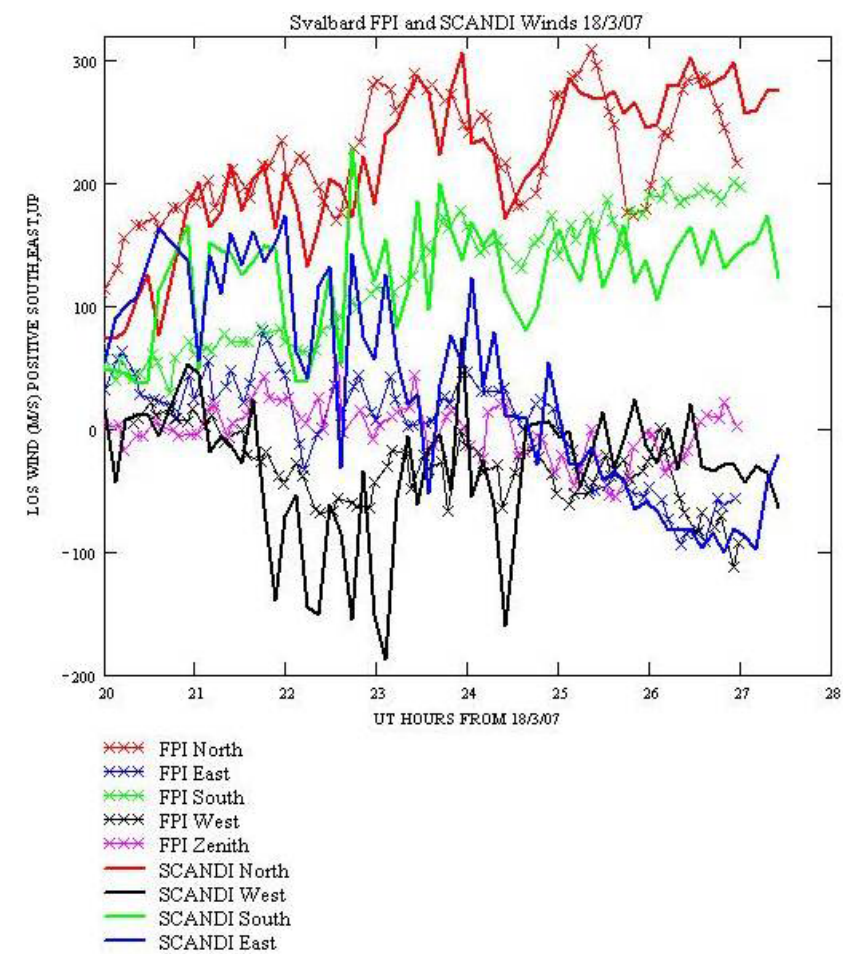

Fig. 16. SCANDI line-of-sight winds on the night of 18 March 2007 show a good match with FPI los winds from corresponding look directions. However, clear spatial structure may be seen in the wind field with a scale-size of the order of around $100 \mathrm{~km}$.

directions to the north, east, south and west. There is an excellent correspondence which confirms that the sky mapping is correct. The figure shows clearly that there was a great deal of structure across the field-of-view, which has a radius of nearly $500 \mathrm{~km}$. In particular there was a large intensity gradient in the meridional direction throughout the night. The intensities to the south were half those to the north except during periods of particle precipitation/auroral arcs when there were sharp short-lived increases by several times the background level. The intensity gradient in the zonal direction was also considerable and changed from higher intensities to the west before 22:00 UT to higher in the east afterwards. These differences are all indicative of the SCANDI field-ofview crossing boundaries between the polar cap and auroral oval, which represent the mapping of the magnetosphere onto the ionosphere.

Figure 16 shows a comparison of SCANDI line-of-sight winds (lines) with the corresponding FPI line-of-sight winds (lines with crosses) for the night of 18-19 March 2007 in the first winter of observations (2006-2007). There is a good match, though not exact. This is largely because the FPI has a $1^{\circ}$ field-of-view, while each SCANDI sector has a solid angle between $0.14 \mathrm{sr}$ (ring 3 ) to $0.24 \mathrm{sr}$ (centre zone) as shown in Table 2. As a result the SCANDI sector data is an integral of the line-of-sight winds from several azimuth and el- evation angles. The sectors in ring 3 cover a $30^{\circ}$ range in azimuth and $17^{\circ}$ in elevation. This is equivalent to a horizontal range of $296 \mathrm{~km}$ azimuthally and $285 \mathrm{~km}$ radially (see Fig. 4a). It is however, possible to construct a wind field by fitting functions to all the line-of-sight wind measurements within a single ring and then deconstruct back to a wind vector that is representative of that sector. This has been described in Sect. 2.4. Note that from the second winter onwards, the SCANDI sectors were divided to provide equal projected zone areas as shown in Fig. $4 \mathrm{c}$ and Table 3.

If the assumption of a uniform wind is correct then the line-of-sight winds to the north should be the reverse of those to the south (i.e., a blue shift in the $630 \mathrm{~nm}$ emission observed to the north should be seen as a red shift of the same magnitude to the south), similarly for the east compared with the west measurements. This is clearly not true and spatial structure in the wind field over the whole field-of-view can be seen in Fig. 16. The main feature for this night is a large meridional wind component varying between $200-300 \mathrm{~m} / \mathrm{s}$ between 21:00-03:00 UT in the north look direction but only rising gradually from $60 \mathrm{~m} / \mathrm{s}$ to $200 \mathrm{~m} / \mathrm{s}$ over the same time period in the south look direction. This results in a large meridional wind gradient. A smaller wind gradient of maximum $50 \mathrm{~m} / \mathrm{s}$ exists in the zonal component, with larger eastward winds seen to the east though the intensities are lower as shown in Fig. 15. After around 22:30 UT the intensity in the east grows larger than all directions except the north. After 01:00 UT the zonal winds accelerate in both the east and west look directions to reach a peak of $100 \mathrm{~m} / \mathrm{s}$ westwards by 03:00 UT. For this night the FPI wind error was around $\pm 10 \mathrm{~m} / \mathrm{s}$, while the SCANDI wind error was about $\pm 15 \mathrm{~m} / \mathrm{s}$, which means that the two instruments generally agree within the limits of their error bars except when the intensities are low (e.g. in the west direction between $\sim 22: 00-23: 00$ UT) or when there may be some underlying meso-scale structure that the SCANDI zone may have smoothed out compared with the $1^{\circ}$ FPI FOV (e.g. in the south after $\sim 02: 00 \mathrm{UT}$ ). Throughout this first winter of observations the correspondence between the SCANDI and FPI LOS winds has been similarly good.

\subsection{SCANDI-ESR radar comparison}

Figure 1 shows the location of the ESR radar beams shown as red crosses superposed on the SCANDI sectors at an altitude of $250 \mathrm{~km}$ for the first radar campaign during 2-4 March 2007. The $42 \mathrm{~m}$ dish at E1 was field-aligned. In order to maximise the time resolution of a spatial scan by the radar, only 3 beam directions were used for the $32 \mathrm{~m}$ dish as described in Sect. 3.3.1.

\subsubsection{Comparison of SCANDI $T_{n}$ with ESR $T_{i}$}

Figure 17 shows a comparison of the SCANDI neutral temperatures with the ESR ion temperatures. The 


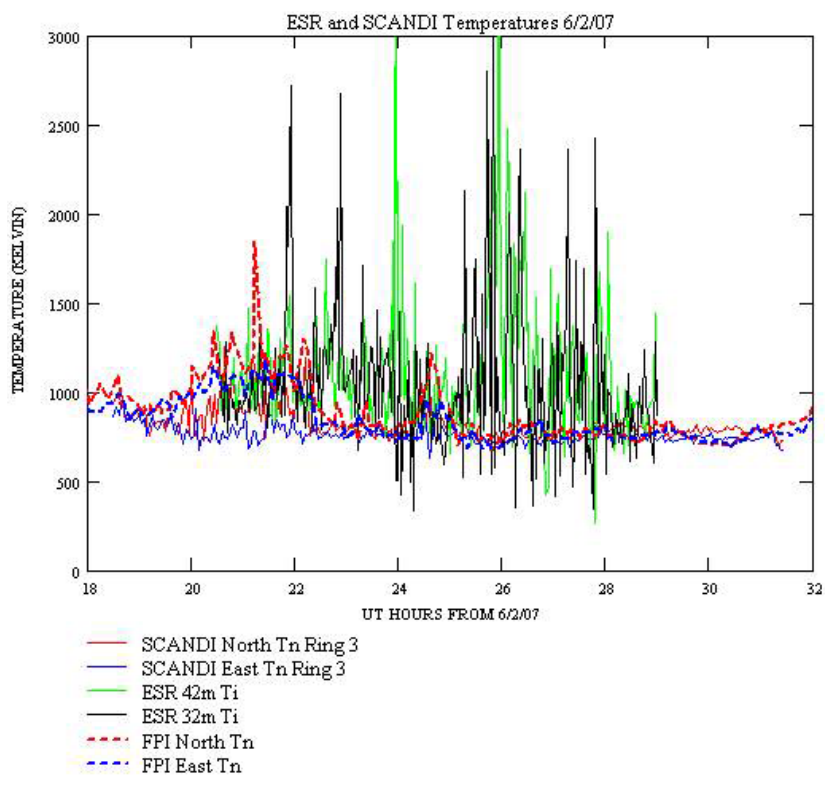

Fig. 17. Comparison of ion temperatures observed by the 2 ESR radars with the neutral temperatures observed by SCANDI on the night of 6-7 February 2007.

ESR measurement of the ion temperatures using the $42 \mathrm{~m}$ dish (green) and $32 \mathrm{~m}$ dish (black) are overlaid. The $42 \mathrm{~m}$ dish was field-aligned, and the $32 \mathrm{~m}$ dish was set on a CP2 scan, with 3 pointing directions: azimuth $=144.0^{\circ}$, elevation $=66.7^{\circ}$; azimuth $=171.6^{\circ}$, elevation $=90.0^{\circ} ;$ azimuth $=171.6^{\circ}$, elevation $=63.2^{\circ}$. The ESR beams at $246 \mathrm{~km}$ altitude map to the $1 \mathrm{st}$ ring of SCANDI, but here the outermost 3rd ring sectors, north (red) and south (blue), are used for comparison in order to allow confirmation of the absolute $T_{n}$ by comparison with the corresponding FPI measurements. The SCANDI neutral temperatures provide a lower boundary to the ion temperatures throughout the night, which provides independent confirmation that the SCANDI temperature analysis is consistent with energy conservation because $T_{i}$ should equal $T_{n}$ when there is no input of magnetospheric energy.

\subsubsection{Case studies of highly localised structure using SCANDI}

The all-sky view given by SCANDI allows studies to show the response of the thermosphere to highly localised regions of particle precipitation, Joule heating and ion drag. Case studies for the nights 15 March 2007 and 4 December 2007 are described in detail in Griffin et al. (2008, 2009). In these papers the ionosphere over Svalbard is probed using the IMAGE magnetometer chain and CUTLASS radars to reveal the horizontal spatial distribution of a highly localised disturbance, and the ESR radars to show the vertical and temporal structure at high resolution. The SCANDI shows thermospheric responses with temperature rises of $100-200 \mathrm{~K}$ over tens of minutes and short-lived bursts of high winds over a range of only a few hundred kilometres. The unexpectedly rapid and localised response of the thermosphere moderates the re-distribution of magnetospheric energy at high latitudes between heating and acceleration of the neutral gas. These examples are strong advocates for the importance of studying the meso-scale response of the thermosphere.

\section{Summary}

The SCANDI is a powerful instrument for investigating the mesoscale structure of the thermosphere on scale sizes of a few hundred kilometres. This paper presents the careful calibration and data analysis required and summarises examples of large wind and temperature gradients that have been observed frequently during a 3 winters of observation. A co-located FPI has provided corroboration of SCANDI measurements of winds and neutral temperatures and a satisfying comparison has been made with radar measurements of ion temperatures. It is well-known that the auroral ionosphere shows much spatial and temporal structure. SCANDI-ESR experiments have shown correspondence between thermospheric and ionospheric parameters on similar scales which are detailed in associated papers. This promotes a need to increase the spatial scale-size of upper atmosphere models to allow for the extent of ion-neutral coupling seen empirically.

\section{Appendix A}

\section{Derivation of the solid angle and area projected on the sky}

The solid angle of a cone or a spherical cap $\Omega$ is given by Eq. (A1).

$\Omega=2 \pi(1-\cos \theta)$

This is applicable to the SCANDI central zone where $\theta$ is the zone's zenith angle.

A more general equation for the solid angles of all the SCANDI zones can be written as Eq. (A2).

$\Omega=2 \pi\left(\cos \theta_{1}-\cos \theta_{2}\right) /_{N}$

Where $\theta_{1}$ and $\theta_{2}$ are the inner and outer zenith angles, respectively, for a SCANDI ring, and $N$ is the number of zones in the ring.

The projected elemental area of a zone on the flat sky $d S$ is given by Eq. (A3).

$d S=x d \theta d x$

Therefore the total projected area $S$ becomes Eq. (A4).

$S=\int_{\theta_{1}}^{\theta_{2}} \int_{x_{1}}^{x_{2}} d S$ 
$S=\frac{\pi h^{2}\left(\tan ^{2} \theta_{2}-\tan ^{2} \theta_{1}\right)}{N}$

Acknowledgements. The authors thank the staff at the Kjell Henriksen Observatory for logistical support. The keograms come from the digital all-sky cameras maintained by the MIRACLE network which is an international collaboration under the leadership of the Finnish Meteorological Institute. EISCAT is an international association supported by research organisations in China (CRIRP), Finland (SA), France (CNRS, till end 2006), Germany (DFG), Japan (NIPR and STEL), Norway (NFR), Sweden (VR), and the United Kingdom (STFC). SCANDI was funded by grant PP/C50165X/1.

Topical Editor M. Pinnock thanks one anonymous referee for her/his help in evaluating this paper.

\section{References}

Aruliah, A. L., Griffin, E. M., Aylward, A. D., Ford, E. A. K., Kosch, M. J., Davis, C. J., Howells, V. S. C., Pryse, S. E., Middleton, H. R., and Jussila, J.: First direct evidence of meso-scale variability on ion-neutral dynamics using co-located tristatic FPIs and EISCAT radar in Northern Scandinavia, Ann. Geophys., 23, 147-162, 2005,

http://www.ann-geophys.net/23/147/2005/.

Batten, S. and Rees, D.: Thermospheric Winds in the Auroral Oval: Observations of Small Scale Structures and Rapid Fluctuations by a Doppler Imaging System, Planet. Space Sci., 38, 675-694, 1990.

Biondi, M. A.: Measured Vertical Motion and Converging and Diverging Horizontal Flow of the Midlatitude Thermosphere, J. Geophys. Res., 11, 84-87, 1984.

Biondi, M. A., Zipf, M. E., Sipler, D. W., and Baumgardner, J. L.: All-Sky Doppler Interferometer for thermospheric dynamics studies, Appl. Optics, 34, 1647-1654, 1995.

Burnside, R. G., Herrero, F. A., Meriwether Jr., J. W., and Walker, J. C. G.: Optical Observations of Thermospheric Dynamics at Arecibo, J. Geophys. Res., 86, 5532-5540, 1981.

Conde, M. and Smith, R. W.: Phase compensation of a separation scanned, all-sky imaging Fabry-Perot spectrometer for auroral studies, Appl. Optics, 36, 5441-5450, 1997.

Conde, M., Crave, J. D., Immel, T., Hoch, E., Stenbaek-Nielson, H., Hallinan, T., Smith, R. W., Olson, J., Wei Sun, Frank, L. A., and Sigwarth, J.: Assimilated observations of thermospheric winds, the aurora, and ionospheric currents over Alaska, J. Geophys. Res., 106, 10493-10508, 2001.

Fuller-Rowell, T. J. and Rees, D.: A Three-Dimensional TimeDependent Global Model of the Thermosphere, J. Atmos. Sci., 37, 2545-2567, 1980.

Greenaway, A. H., Lyons, A., McWhirter, I., and Rees, D.: Miniature Imaging Photon Detector, SPIE, 31, pp 365-367, 1982.

Greet, P. A., Conde, M. G., Dyson, P. L., Innis, J. L., Breed, A. M., and Murphy, D. J.: Thermospheric wind field over Mawson and Davis, Antarctica: simultaneous observations by two FabryPerot spectrometers of $630 \mathrm{~nm}$ emission, J. Atmos. Solar Terr. Phys., 61, 1025-1045, 1999.
Griffin, E. M., Mller-Wodarg, I. C. F., Aruliah, A., and Aylward, A.: Comparison of high-latitude thermospheric meridionalwinds I: optical and radar experimental comparisons, Ann. Geophys., 22, 849-862, 2004, http://www.ann-geophys.net/22/849/2004/.

Griffin, E. M., Aruliah, A. L., McWhirter, I., Yiu, H.-C. I., Charalambous, A., and McCrea, I.: Upper thermospheric neutral wind and temperature measurements from an extended spatial field, Ann. Geophys., 26, 2649-2655, 2008, http://www.ann-geophys.net/26/2649/2008/.

Griffin, E. M., Aruliah, A. L., McWhirter, I., Yiu, H.-C. I., and Charalambous, A.: Upper thermospheric ion-neutral coupling from combined optical and radar experiments over Svalbard, Ann. Geophys., 27, 4293-4303, 2009, http://www.ann-geophys.net/27/4293/2009/.

Holmes, J. M., Conde, M., Deehr, C., and Lummerzheim, D.: Morphology of evening sector aurorae in L557.7-nm Doppler temperatures, Geophys. Res. Lett., 32, L02103, doi:10.1029/2004GL021553, 2005.

IDL Online Help: MAP_SET, Map projections, Azimuthal Equidistant Projection, IDL Reference Guide, IDL Version 6.3, April 2006 Edition, Copyright (C) RSI, All Rights Reserved, 1 March 2006.

Kumler, J. and Bauer, M. L.: Fish-eye lens designs and their relative performance, Proc. SPIE, 4093, 360, 2000.

McWhirter, I., Rees, D., and Greenaway, A. H.: Miniature Imaging Photon Detectors III. An Assessment of the Performance of the Resistive Anode IPD, J. Phys. E.: Sci.Instrum., 15, 145-150, 1982.

McWhirter, I.: Electron Multiplying CCDs - New Technology for Low Light Level Imaging, Proceedings of 33rd Annual European Meeting on Atmospheric Studies by Optical Methods, IRF Sci. Rep., 292, 63-68, http://www.irf.se/publications/ proc33AM/mcwhirter.pdf, 2008

Meriwether, J. W., Tepley, C. A., Price, S. A., and Hays, P. B.: Remote Ground-Based Observations of Terrestrial Airglow Emissions and Thermospheric Dynamics at Calgary, Alberta, Canada, Optical Eng., 22, 128-131, 1983.

Rees, D., McWhirter, I., Rounce, P. A., Barlow, F. E., and Kellock, S. J.: Miniature Imaging Photon Detectors, J. Phys. E.: Sci.Instrum., 13, 763-770, 1980.

Rees, D., McWhirter, I., Hays, P. B., and Dines, T.: A Stable, Rugged, Capacitance-Stabilised Piezoelectric Scanned FabryPerot Etalon, J. Phys. E: Sci. Instrum, 14, 1320-1325, 1981.

Rees, D., Greenaway, A. H., Gordon, R., McWhirter, I., Charleton, P. J., and Steen, A.: The Doppler Imaging System: Initial Observations of the Auroral Thermosphere, Planet. Space Sci., 32, 273-285, 1984.

Rishbeth, H. and Garriott, O. K.: Introduction to Ionospheric Physics, Academic Press, 1969.

Roble, R. G., Dickinson, R. E., Ridley, E. C., Emery, B. A., Hays, P. B., Killeen, T. L., and Spencer, N. W.: The High Latitude Circulation and Temperature Structure of the Thermosphere Near Solstice, Planet. Space Sci., 31, 1479-1499, 1983.

Thoby, M.: Fisheye lenses compared: Sigma $8 \mathrm{~mm}$ $\mathrm{f} / 4$ Vs Nikkor $10,5 \mathrm{~mm} \quad \mathrm{f} / 2,8$. http://michel.thoby. free.fr/Blur_Panorama/Nikkor10-5mm_or_Sigma8mm/ sigma_or_Nikkor/Comparison_short_version_Eng.html, 2006. 